\title{
Systematic Construction of Higher Order Bases for the Finite Element Analysis of Multiscale Elliptic Problems
}

\author{
Soheil Soghrati ${ }^{1}$ and Ilinca Stanciulescu, ${ }^{2, *}$ \\ ${ }^{1}$ University of Illinois at Urbana-Champaign, Urbana, IL 61801, U.S.A. \\ ${ }^{2}$ Rice University, Houston, TX, 77005, U.S.A. \\ ${ }^{*}$ Corresponding Author, ilinca@rice.edu
}

\begin{abstract}
We introduce a new approach to derive higher order basis functions implemented in the Multiscale Finite Element Method (MsFEM) for elliptic problems. MsFEM relies on capturing small scale features of the system through bases utilized in the coarse scale solution. The proposed technique for the derivation of such bases is completely systematic and the increase in the associated computational cost is insignificant. We also show that the implementation of higher order bases in MsFEM leads to the similar advantages as using higher order Lagrangian shape functions in the conventional finite element method. Various numerical examples for heat transfer problems with periodic or heterogeneous thermal properties are studied to shw the efficiency and improved characteristics of the proposed higher order bases.
\end{abstract}

\section{Introduction}

Second order elliptic differential equations with multiple-scale features appear in variety of materials science and engineering problems, including heat conduction in heterogeneous materials and flow in porous media $[1,2]$. In such phenomena, variations and fluctuations of material properties at the fine scale have a significant impact on the behavior of the system at the coarse level. Obtaining an accurate solution for such problems via the standard finite element method (FEM) requires meshes that resolve the smallest scale of the system, which often leads to extremely large numbers of degrees of freedom. Hence, the large size of the resulting discrete problem considerably increases the computational cost, which at best leads to long running times but in many cases exceeds available memory capacities. While the parallel implementation alleviates the limitations in the computational resources, it can not reduce the size of the discrete problem.

To address the problems discussed above, several techniques including those based on the homogenization theory [3] and upscaling methods $[4,5]$ have emerged to determine the effective properties of the system and incorporate them in the coarse-scale simulation. Other approaches presented in the literature aim at developing multiscale analysis methods by incorporating the fine scale information in the coarse scale solution. Hughes et. al $[6,7]$ proposed a variational multiscale method (VMM) in which the solution is evaluated at two steps: the non-numerical solution of the fine 
scale and its implementation for solving the coarse scale problem. Babuska et al [8] developed a generalized finite element method with special non-polynomial basis functions for approximating second-order elliptical problems. In the heterogeneous multiscale method (HMM), introduced by Engquist et. al [9, 10], different scales of the system are separated through solving the macroproblem and evaluating the missing information by performing local analyses at finer scales.

The Multiscale Finite Element Method (MsFEM) is among the most successful approaches for solving elliptic problems with oscillatory coefficients of governing equations $[11,12,13]$. The main idea in this method is to transfer the effect of the fine scale properties to the coarse level by replacing the Lagrangian shape functions with appropriate bases that carry out these information. A great advantage of the method is that it decouples the calculation of the basis functions in different elements, which suites the parallel analysis implementation. One of the major issue in MsFEM, however, is the slow rate of convergence due to the resonance between the grid size and the physical scale of the problem. This leads to a mismatch between the fine and coarse scale solutions over element boundaries. To reduce the resonance error, an oversampling technique is proposed in [11], where the sampling is performed over a larger domain and interior data are used to evaluate the basis functions. Reference [14] presents a comprehensive overview on recent multiscale methods including MsFEM and VMM for second-order elliptic problems.

The algorithm presented in [11] for evaluating MsFEM basis functions is limited to modifying standard linear or bilinear shape functions. The extension to higher order bases is the next step, where among previous related efforts we can mention the work by Allaire and Brizzi [15]. However, the method presented in [15] for evaluating higher order bases is mathematically complex and the desired results are achieved at a higher computational cost. In the current paper, we introduce a simpler and yet generalized algorithm for constructing higher order bases that is applicable to elements with any desired configuration and number of nodes. We also show that while the proposed higher order bases considerably improve the accuracy and rate of convergence, such improvements are obtained without significant increase in the computational cost.

The remainder of the paper is organized as follows. In section 2, we present the elliptic multiscale problem and briefly explain the original MsFEM formulation in [11]. In section 3, the proposed derivation of higher order MsFEM basis functions is presented. We first introduce a systematic approach for developing basis functions for 1D elements and then show how the resulting1D bases can be employed for constructing 2D basis functions. In section four, we analyze the computational cost, accuracy and the rate of convergence of these elements by studying several heat transfer example problems with periodic or heterogeneous thermal properties.

\section{Second Order Elliptic Problems and MsFEM}

Consider an open domain $\Omega \subset \mathbb{R}^{n}$ and its boundary $\Gamma=\bar{\Omega} \backslash \Omega=\Gamma_{u} \cup \Gamma_{q}$ with outward unit normal n. Let $g(\mathbf{x}): \boldsymbol{\Gamma}_{\mathbf{u}} \rightarrow \mathbb{R}$ and $h(\mathbf{x}): \boldsymbol{\Gamma}_{\mathbf{q}} \rightarrow \mathbb{R}$ define the Dirichlet and Neumann boundary conditions for this system, respectively. Given the positive definite tensor $\mathbf{a}(\mathbf{x}): \Gamma_{q} \rightarrow \mathbb{R}^{n} \times \mathbb{R}^{n}$ (which, for instance, consists of thermal conductivity coefficients for heat transfer problems), the second-order elliptic equation studied in this work is expressed as 


$$
\begin{array}{ccccc}
-\nabla \cdot(\mathbf{a}(\mathbf{x}) \nabla u) & = & f & \text { in } & \Omega \\
u & = & g(\mathbf{x}) & \text { on } \Gamma_{u}, \\
\mathbf{a}(\mathbf{x}) \nabla u \cdot \mathbf{n} & = & h(\mathbf{x}) & \text { on } \Gamma_{q} .
\end{array}
$$

The weak form of Eq. (2.1) is written as: Find $u \in \mathcal{U}$ such that

$$
\int_{\Omega} \mathbf{a}(\mathbf{x}) \nabla u \nabla v d \Omega=\int_{\Omega} f v d \Omega+\int_{\Gamma_{q}} g(\mathbf{x}) v d \Gamma \quad \forall v \in \mathcal{V},
$$

where $\mathcal{U}=\left\{u(\mathbf{x}),\left.u\right|_{\Gamma_{u}}=g(\mathbf{x})\right\} \subset H^{1}(\bar{\Omega})$ and $\mathcal{V}=\left\{v(\mathbf{x}),\left.v\right|_{\Gamma_{u}}=0\right\} \subset H_{0}^{1}(\bar{\Omega})$ are the solution and weight function spaces, respectively. Eq. (2.2) can be used to derive the Galerkin approximation and obtain the associated FEM formulation. However, an FEM solution requires highly-refined meshes to resolve the smallest scale of the problem, which can be extremely expensive if $\mathbf{a}(\mathbf{x})$ is very heterogeneous or highly oscillatory. MsFEM addresses this issue by evaluating basis functions that capture the small scale effects within a coarse finite element mesh. To evaluate basis function $N_{i}^{(k)}$ corresponding to element $\Omega^{k}$, we solve the homogenized form of Eq. (2.1),

$$
\nabla \cdot\left(\mathbf{a}(\mathbf{x}) \nabla N_{i}^{(k)}\right)=0 \quad \text { in } \quad \Omega^{k} \subset \Omega
$$

subject to appropriate boundary conditions. This requires that the resulting bases satisfy the Kronecker delta property at the element nodes $N_{i}^{(k)}\left(x_{j}\right)=\delta_{i j}$. Determining appropriate boundary conditions for Eq. (2.3) is not trivial; choices made here significantly affect the accuracy of the MsFEM solution. Two choices for the boundary conditions (for four node 2D elements) are proposed in [11]. The first is to consider linear functions, i.e., restrictions to the boundaries of the standard bilinear shape functions. This choice does not lead to satisfactory results because it neglects the oscillations in the fine scale over the boundaries of the coarse mesh. A second option is proposed, where the effect of oscillations in the fine scale properties is included by obtaining the solution $N_{i}^{\Gamma}$

of the reduced form of Eq. (2.3) over each boundary of the course element, $\frac{d}{d x_{\Gamma}}\left(\mathbf{a}_{\Gamma}\left(x_{\Gamma}\right) \frac{d N_{i}^{\Gamma}}{d x_{\Gamma}}\right)=$ 0 on $\Gamma_{i}^{k}=\partial \Omega^{k}$, subject to $N_{i}^{\Gamma}\left(x_{j}\right)=\delta_{i j}$. In the special case where the conductivity tensor is a separable function, $\mathbf{a}(\mathbf{x})=\mathbf{a}_{1}(x) \mathbf{a}_{2}(y)$, the $2 \mathrm{D}$ basis functions can be computed directly from the tensor product of the boundary condition functions. The interested reader can find a detailed description of the MsFEM, including the estimation of the resonance error in $[11,12]$.

\section{Extension to Higher Order Elements}

In this section, we introduce a general approach for evaluating the MsFEM basis functions for higher order elements with different number of nodes. We aim to preserve some of the properties of the Lagrangian shape functions in the higher order MsFEM bases. The approach we propose not only avoids heavy mathematical derivation and the use of homogenization theory in computing the MsFEM bases, but also provides more insight over the features of these bases. We follow a direct extension of the procedure explained in section 2 by solving an FEM problem over the coarse elements subject to appropriate boundary conditions. The boundary conditions associated with 
Eq. (2.3) determine how well the characteristics of the fine scale are captured in the basis and thus transferred to the coarse-scale solution. In order to achieve an acceptable accuracy, we first need to incorporate the fine scale information in the boundary conditions of higher order bases. To achieve this goal, we first devise an approach for evaluating higher order bases for 1D problems, and then employ these 1D-bases as boundary conditions for the 2D basis functions.

\subsection{Higher Order Bases for 1D Problems}

Consider the 1D elliptic problem

$$
\frac{d}{d x}\left(a(x) \frac{d u}{d x}\right)=f(x) \quad \forall x \in[0, L] .
$$

Constructing two-node bases for the MsFEM solution of this problem using the method outlined in section 2 is quite straightforward: two-node 1D basis functions are obtained form the solutions of $\frac{d}{d x}\left(a(x) \frac{d u}{d x}\right)=0 \quad \forall x \in[0, L]$ subject to $N_{i}^{k}\left(x_{j}\right)=\delta_{i j}$ boundary conditions. In order to extend this algorithm to higher order bases, we require that the basis functions are $C^{0}$-continuous and satisfy the partition of unity, i.e., $\sum_{i=1}^{n} N_{i}^{k}=1$.

Before introducing an algorithm for creating higher order 1D basis functions, we study some of the important characteristics of standard Lagrangian shape functions to mimic similar properties in the evaluation of higher order bases. Unlike linear shape functions, higher order Lagrangian shape functions do not satisfy the homogenized form of a second order elliptic equation. Similarly, we see no reason to require higher order multiscale bases to satisfy this equation. The $2^{\text {nd }}$ order Lagrangian shape functions are constructed through the linear combination of $1^{\text {st }}$ order Lagrangian shape functions and a parabolic function. This parabolic function can be the solution of a particular instance of Eq. (3.1) (with constant $a(x)=a_{0}$ ), i.e., $f(x)=1$ and subject to zero essential boundary conditions. Similarly, multiscale bases for three-node elements, $N_{i}^{(3)}$, can be constructed through a linear combination of two-node basis functions, $N_{e}^{(2)}$, and the solution $N_{p}$ of Eq. (3.1) with $f(x)=1$, subject to zero essential boundary conditions. Using $e$ and $i$ notations to refer to degrees of freedom of two-node and higher order elements, respectively, we can express the higher order bases for three-node elements as $N_{i}^{(3)}=\delta_{e i} N_{e}^{(2)}+\alpha_{i} N_{p}$ :

$$
\left\{\begin{array}{l}
N_{1}^{(3)}=N_{1}^{(2)}+\alpha_{1} N_{p} \rightarrow \alpha_{1}=-\left.\left(N_{1}^{(2)} / N_{p}\right)\right|_{\xi=0} \\
N_{2}^{(3)}=\alpha_{2} N_{p} \\
N_{3}^{(3)}=N_{2}^{(2)}+\alpha_{3} N_{p} \rightarrow \alpha_{2}=\left.\left(1 / N_{p}\right)\right|_{\xi=0}=-\left.\left(N_{2}^{(2)} / N_{p}\right)\right|_{\xi=0}
\end{array}\right.
$$

In the expression above, $\alpha_{i}$ are determined such that Kronecker delta condition is satisfied at the mid-point, i.e., $\left.N_{i}^{(3)}\right|_{\xi_{j}}=\delta_{i j}$, where $\xi \in[-1,1]$ are natural coordinates. Figure 1 compares second order standard Lagrangian shape functions with the multiscale basis functions evaluated for the conductivity tensor $a(x)=2000(1.3+\sin 400 \pi x)(1.3+\cos (400 \pi x+2 \pi / 5))$, with five oscillations per coarse element. Note that the resulted bases converges to standard Lagrangian shape functions as oscillations in the fine scale properties are damped out. 

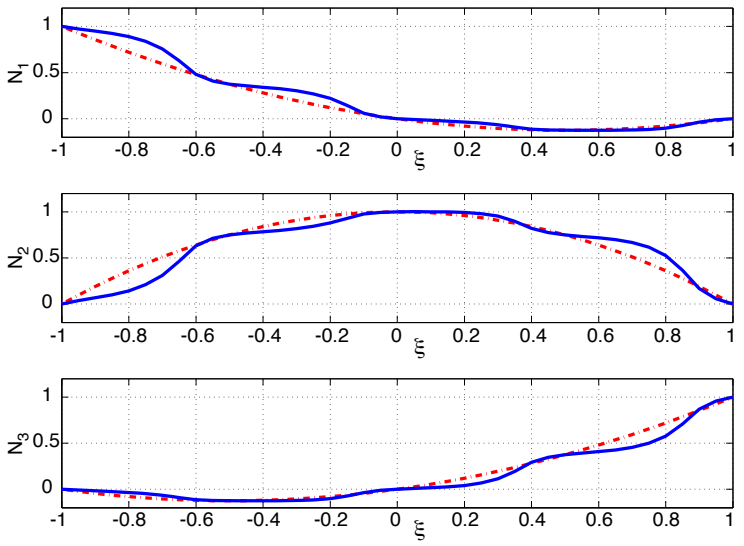

Figure 1: Multiscale (solid lines) and Lagrangian (dotted lines) shape functions

The proposed approach for evaluating the basis functions of three-node elements can be easily extended to even higher order bases. In general, 1D bases for an $n$-node element are expressed as $N_{i}^{(n)}=\delta_{e i} N_{e}^{(2)}+\sum_{j=1}^{n-2} \alpha_{i}^{(j)} N_{p}^{(j)}$, where functions $N_{p}^{(j)}$ are evaluated from solving Eq. (3.1) with the right-hand-side function $f^{(j)}(\xi)=\xi^{j-1}$. Coefficients $\alpha_{i}^{(j)}$ are then computed such that the resulting basis functions satisfy the Kronecker delta property at the interior nodes, i.e., $N_{i}^{(n)}\left(\xi_{k}\right)=\delta_{i k}$.

\subsection{Higher Order Bases for 2D Problems}

The extension of the algorithm described in section 2 to higher order $2 \mathrm{D}$ bases is not difficult provided that good choices are made for boundary conditions of coarse elements. The approach adopted here is to solve Eq. 2.1 over a coarse element with 1D basis functions corresponding to the number of nodes along edges of the element edges as the boundary conditions. The resulting solution can be then used as basis functions for elements with nodes distributed only over the boundaries, i.e., serendipity-type elements. The basis functions calculated with this method for an 8 -node $2 \mathrm{D}$ element are illustrated in figure 2 , where bases of three-node $1 \mathrm{D}$ elements define the boundary conditions along element's edges.
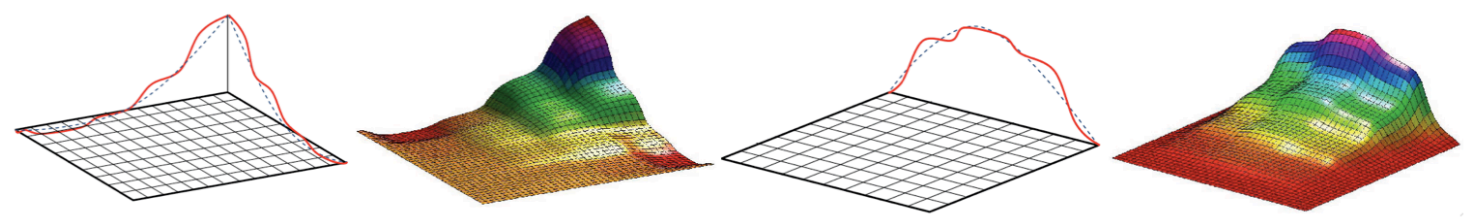

Figure 2: Boundary conditions and resulting MsFEM basis functions for an 8-node element

To construct basis functions for elements with interior nodes, similar to the approach discussed for $1 \mathrm{D}$ elements, we first solve Eq. (2.1) subject to zero boundary conditions and appropriate righthand-side polynomial functions. In general, $2 \mathrm{D}$ bases of a standard $n$-node quadrilateral element with $m$ interior nodes are given by $N_{i}^{(n)}=\delta_{e i} N_{e}^{(n-m)}+\sum_{j=1}^{\sqrt{m}} \sum_{k=1}^{\sqrt{m}} \alpha_{i}^{(j, k)} N_{p}^{(j, k)}$, where $N_{e}^{(n-m)}$ are 
the basis functions of the corresponding serendipity-type element with $n-m$ nodes and subscript $e$ refers to the degrees of freedom in this element. $N_{p}^{(j, k)}$ are evaluated from solving Eq. (2.1) with the right-hand-side function $f^{(j, k)}(\xi, \eta)=\xi^{j-1} \eta^{k-1}$. Coefficient $\alpha_{i}^{(j, k)}$ corresponding to the $i-$ th basis function is determined by satisfying the Kronecker delta property at element's nodes, i.e., $N_{i}^{(n)}(\xi, \eta)_{p}=\delta_{i p}$. Following this algorithm, basis functions for a standard 9-node quadrilateral element, for example, is written as $N_{e}^{(9)}=\delta_{e i} N_{e}^{(8)}+\alpha_{i} N_{p}$, where $N_{p}$ is obtained from solving $-\nabla \cdot\left(\mathbf{a}(\mathbf{x}) \nabla N_{p}\right)=1$ in $\Omega^{k}$ and subject to $N_{p}=0$ on $\partial \Omega^{k}$ as boundary conditions.

If the conductivity tensor is composed of separable functions in the Euclidian space, i.e., $\mathbf{a}(\mathbf{x})=$ $\mathbf{a}_{1}(x) \mathbf{a}_{2}(y)$, we can use a computationally less expensive method: $2 \mathrm{D}$ shape functions are obtained from the tensor product of 1D bases evaluated over the boundaries of the coarse element (figure 3.) It must be noted that the method discussed earlier for evaluating the bases from the numerical solution of Eq. 2.1 and the current approach yield different basis functions. However, the results presented in section 4 will demonstrate that both schemes yield similar level of accuracy in problems with separable conductivity tensors.

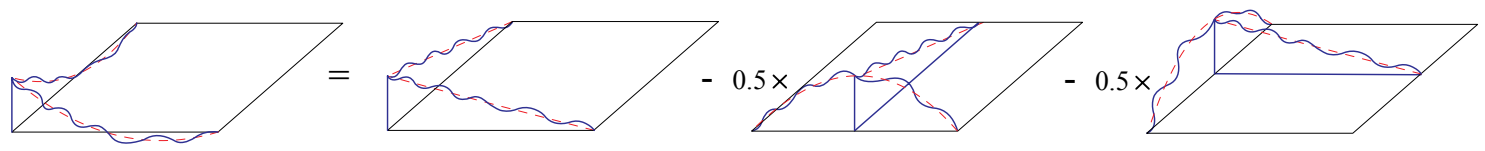

Figure 3: Construction of MsFEM basis functions through the tensor product of 1D bases

\section{Numerical Experiments}

In this section, we study the performance of the proposed higher order bases through several numerical tests, with reference solutions obtained by stanard FEM with extremely refined meshes that resolve the small scale.

Example 1 (1D problem): For 1D problems, MsFEM yields similar results as standard FEM, provided that the product of the number of elements in the fine and course meshes, i.e., $N_{f} \times N_{c}$, is equal to the number of elements utilized in the standard FEM solution. However, the current 1D example problem sheds more light on the behavior of these bases and offers additional insight into the MsFEM algorithm. For these reason, we study the problem: $\frac{d}{d x}\left(a_{0}\left(1.3+\sin \left(\frac{2 \pi x}{\varepsilon}\right)\right)\left(1.3+\cos \left(\frac{2 \pi x}{\varepsilon}+\frac{2 \pi}{5}\right)\right) \cdot \frac{d u}{d x}\right)=-1000 \forall x \in[0, L]$, where $u(0)=u(L)=0$, and $\varepsilon=0.025$ characterizes the small scale properties (40 oscillations per unit length of the domain). The length of the domain is $L=5$ and the coarse mesh composed of $N_{c}=100$ nodes.

Table 1 shows the performance of the MsFEM solution. The two cases correspond to utilizing linear and second-order Lagrangian shape functions for evaluating the fine scale solution. Values of the $l^{2}$-norm of the error for this problem are exactly the same for all coarse scale elements $(2,3$ or 4-node) presented in this Table 1 . At the first glance, this might indicate the low performance of higher order bases: despite the additional cost imposed to the solution, higher order bases do not improve the convergence of MsFEM. However, this behavior can be easily explained. MsFEM is the generalization of the standard FEM through modifying shape functions to include the small scale information. Accordingly, the behavior of MsFEM bases is similar to that of Lagrangian 
shape functions of the same order in the standard FEM for the same problem but with a constant conductivity tensor. Employing any Lagrangian shape functions for solving a 1D elliptic problem with constant conductivity tensor through the standard FEM yields super-convergent results for the nodal values. Similarly, higher order bases in MsFEM yield the exact same nodal solution at the common nodes of coarse meshes used to discretize the domain.

\begin{tabular}{|c|c|c|c|}
\hline \multicolumn{2}{|c|}{$1^{\text {st }}$ order } & \multicolumn{2}{c|}{$2^{\text {nd }}$ order } \\
\hline$N_{f}$ & $\|E\|_{l^{2}}$ & $N_{f}$ & $\|E\|_{l^{2}}$ \\
\hline \hline 100 & $9.163 \cdot 10^{-4}$ & 10 & $8.723 \cdot 10^{-3}$ \\
\hline 200 & $2.287 \cdot 10^{-4}$ & 20 & $6.218 \cdot 10^{-4}$ \\
\hline 300 & $1.012 \cdot 10^{-4}$ & 30 & $9.914 \cdot 10^{-5}$ \\
\hline 400 & $5.660 \cdot 10^{-5}$ & 40 & $3.179 \cdot 10^{-5}$ \\
\hline 500 & $3.589 \cdot 10^{-5}$ & 50 & $1.266 \cdot 10^{-5}$ \\
\hline 600 & $2.467 \cdot 10^{-5}$ & 60 & $5.692 \cdot 10^{-6}$ \\
\hline 700 & $1.789 \cdot 10^{-5}$ & 70 & $2.689 \cdot 10^{-6}$ \\
\hline 800 & $1.350 \cdot 10^{-5}$ & 80 & $1.226 \cdot 10^{-6}$ \\
\hline
\end{tabular}

Table 1: MsFEM Results (Example 1)

\begin{tabular}{|c|c|c|c|c|c|}
\hline \multicolumn{2}{|c|}{ Two-scaled } & \multicolumn{3}{c|}{ Three-scaled solution } & \\
\hline$N_{f}$ & $\|E\|_{l^{2}}$ & $N_{f 1}$ & $N_{f 2}$ & $\|E\|_{l^{2}}$ & Nodes \\
\hline \hline 100 & $9.163 \cdot 10^{-4}$ & 10 & 5 & $5.219 \cdot 10^{-4}$ & 10000 \\
\hline 200 & $2.287 \cdot 10^{-4}$ & 20 & 5 & $8.220 \cdot 10^{-5}$ & 20000 \\
\hline 300 & $1.012 \cdot 10^{-4}$ & 30 & 5 & $5.012 \cdot 10^{-5}$ & 30000 \\
\hline 400 & $5.660 \cdot 10^{-5}$ & 40 & 5 & $2.815 \cdot 10^{-5}$ & 40000 \\
\hline 500 & $3.589 \cdot 10^{-5}$ & 25 & 10 & $\mathbf{9 . 4 1 1 \cdot 1 0 ^ { - 6 }}$ & 50000 \\
\hline 600 & $2.467 \cdot 10^{-5}$ & 30 & 10 & $7.215 \cdot 10^{-6}$ & 60000 \\
\hline 700 & $1.789 \cdot 10^{-5}$ & 35 & 10 & $5.076 \cdot 10^{-6}$ & 70000 \\
\hline 800 & $\mathbf{1 . 3 5 0} \cdot 10^{-5}$ & 40 & 10 & $3.128 \cdot 10^{-6}$ & 80000 \\
\hline
\end{tabular}

Table 2: Two and three scaled solutions (Example 1)

The main advantage of higher order 1D bases appears in problems with more than two scales. The order of the Lagrangian shape functions used in the solution at the small scale has a crucial effect on the accuracy. The reason can be traced back to the effect of the oscillations in the small scale properties which prevents the standard FEM from yielding super-convergent results. In this case, as shown in Table 1, using $2^{\text {nd }}$ order Lagrangian shape functions for the solution at the small scale yields a better accuracy per computation cost than linear functions. For instance, 50 secondorder elements in the small scale provide better accuracy than 800 linear elements (the size of the resulted stiffness matrix is therefore reduced by a factor of 8 when second order elements are used.)

The same characteristic can be used to further reduce the size

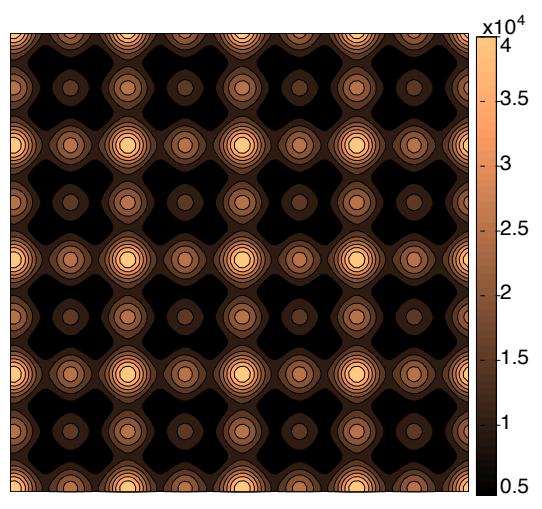

Figure 4: Oscillations in the conductivity tensor (Example 2)

$a_{0}\left(1.3+\sin \frac{2 \pi x}{\varepsilon}\right)\left(1.3+\sin \frac{2 \pi y}{\varepsilon}\right)$ corresponds to 60 oscillations in each direction over the domain (figure 4). The MsFEM solutions of the discrete model in MsFEM. For instance, we can solve the current problem in three scales, using linear shape functions in the finest scale, three-node bases in the small scale and 2-node bases in the coarse mesh. In this case, we expect to achieve more accurate results compared to the solution obtained if only two scales with linear and modified linear shape functions are used. Table 2 confirms the expectation: while the total number of degrees of freedom is kept constant in each row, the three-scaled solution described above yields more accurate results. Note that using three scales for solving this problem is only a choice for the numerical solution and does not correspond to the physical scales of the problem.

Example 2 (periodic separable conductivity tensor): In this example problem, we solve Eq. (2.1) for $a(\mathbf{x})=$ $\left(1.3+\cos \left(\frac{2 \pi x}{\varepsilon}+\frac{2 \pi}{5}\right)\right)\left(1.3+\cos \left(\frac{2 \pi y}{\varepsilon}+\frac{2 \pi}{5}\right)\right)$ and $f=-1000$ over 
using 4 and 9-node elements (using the tensor product approach) are reported in Table 3 and the convergence in $l^{2}$-norm of the error is illustrated in Figures 5 and 6 . Note that for clarity the range of error in the two graphs differs by a factor of 10. The accuracy of the MsFEM solution is limited by the size of the coarse mesh, which is independent of the resolution of the discretization in the fine scale. Using $10 \times 10$ or $20 \times 20$ coarse meshes with modified bilinear shape functions, i.e., 4-node elements, does not provide results with a satisfactory level of accuracy, even for extremely refined meshes in the fine scale. As shown in Table 3, the $l^{2}$-norm norm of the error in this case is approximately 10 times larger than the error obtained with 9-node basis functions.

\begin{tabular}{|c|c||c|c||c|c|}
\hline \multicolumn{2}{|c||}{ Mesh } & \multicolumn{2}{c||}{ 4-node elements } & \multicolumn{2}{c|}{ 9-node elements } \\
\hline$N_{c}$ & $N_{f}$ & $E_{l^{2}}$ & $E_{\infty}$ & $E_{l^{2}}$ & $E_{\infty}$ \\
\hline 10 & 10 & $1.986 \cdot 10^{-1}$ & $3.550 \cdot 10^{-1}$ & $1.372 \cdot 10^{-2}$ & $2.452 \cdot 10^{-2}$ \\
\hline 10 & 20 & $8.521 \cdot 10^{-2}$ & $1.525 \cdot 10^{-1}$ & $6.958 \cdot 10^{-4}$ & $9.512 \cdot 10^{-4}$ \\
\hline 10 & 30 & $3.489 \cdot 10^{-2}$ & $6.110 \cdot 10^{-2}$ & $4.561 \cdot 10^{-4}$ & $7.308 \cdot 10^{-4}$ \\
\hline 10 & 40 & $2.220 \cdot 10^{-2}$ & $4.010 \cdot 10^{-2}$ & $3.961 \cdot 10^{-4}$ & $6.149 \cdot 10^{-4}$ \\
\hline 10 & 50 & $1.286 \cdot 10^{-2}$ & $2.343 \cdot 10^{-2}$ & $3.186 \cdot 10^{-4}$ & $5.851 \cdot 10^{-4}$ \\
\hline 20 & 10 & $8.825 \cdot 10^{-2}$ & $1.576 \cdot 10^{-1}$ & $4.599 \cdot 10^{-4}$ & $7.484 \cdot 10^{-4}$ \\
\hline 20 & 20 & $2.564 \cdot 10^{-2}$ & $4.588 \cdot 10^{-2}$ & $3.030 \cdot 10^{-4}$ & $5.538 \cdot 10^{-4}$ \\
\hline 20 & 30 & $1.073 \cdot 10^{-2}$ & $1.924 \cdot 10^{-2}$ & $2.146 \cdot 10^{-4}$ & $3.958 \cdot 10^{-4}$ \\
\hline 20 & 40 & $5.744 \cdot 10^{-3}$ & $1.029 \cdot 10^{-2}$ & $2.343 \cdot 10^{-4}$ & $4.098 \cdot 10^{-4}$ \\
\hline 20 & 50 & $3.109 \cdot 10^{-3}$ & $5.720 \cdot 10^{-3}$ & $2.342 \cdot 10^{-4}$ & $4.088 \cdot 10^{-4}$ \\
\hline
\end{tabular}

\begin{tabular}{|c|c||c|c||c|c|}
\hline \multicolumn{2}{|c||}{ Mesh } & \multicolumn{2}{c||}{ 4-node elements } & \multicolumn{2}{c|}{ 9-node elements } \\
\hline$N_{c}$ & $N_{f}$ & $E_{l^{2}}$ & $E_{\infty}$ & $E_{l^{2}}$ & $E_{\infty}$ \\
\hline 30 & 10 & $3.786 \cdot 10^{-2}$ & $6.772 \cdot 10^{-2}$ & $8.219 \cdot 10^{-4}$ & $1.420 \cdot 10^{-3}$ \\
\hline 30 & 20 & $1.134 \cdot 10^{-2}$ & $2.028 \cdot 10^{-2}$ & $3.155 \cdot 10^{-4}$ & $4.968 \cdot 10^{-4}$ \\
\hline 30 & 30 & $4.820 \cdot 10^{-3}$ & $8.720 \cdot 10^{-3}$ & $2.380 \cdot 10^{-4}$ & $4.153 \cdot 10^{-4}$ \\
\hline 30 & 40 & $2.463 \cdot 10^{-3}$ & $4.381 \cdot 10^{-3}$ & $2.350 \cdot 10^{-4}$ & $4.149 \cdot 10^{-4}$ \\
\hline 30 & 50 & $1.206 \cdot 10^{-3}$ & $2.341 \cdot 10^{-3}$ & $2.350 \cdot 10^{-4}$ & $4.149 \cdot 10^{-4}$ \\
\hline 60 & 10 & $1.173 \cdot 10^{-2}$ & $2.094 \cdot 10^{-2}$ & $3.475 \cdot 10^{-4}$ & $6.247 \cdot 10^{-4}$ \\
\hline 60 & 20 & $2.781 \cdot 10^{-3}$ & $5.038 \cdot 10^{-3}$ & $2.266 \cdot 10^{-4}$ & $4.087 \cdot 10^{-4}$ \\
\hline 60 & 30 & $1.031 \cdot 10^{-3}$ & $1.793 \cdot 10^{-3}$ & $2.266 \cdot 10^{-4}$ & $4.087 \cdot 10^{-4}$ \\
\hline 60 & 40 & $3.909 \cdot 10^{-4}$ & $7.074 \cdot 10^{-4}$ & $2.266 \cdot 10^{-4}$ & $4.087 \cdot 10^{-4}$ \\
\hline 60 & 50 & $2.549 \cdot 10^{-4}$ & $4.277 \cdot 10^{-4}$ & $2.266 \cdot 10^{-4}$ & $4.087 \cdot 10^{-4}$ \\
\hline
\end{tabular}

Table 3: Example 2 (periodic separable conductivity tensor), performance of higher order elements

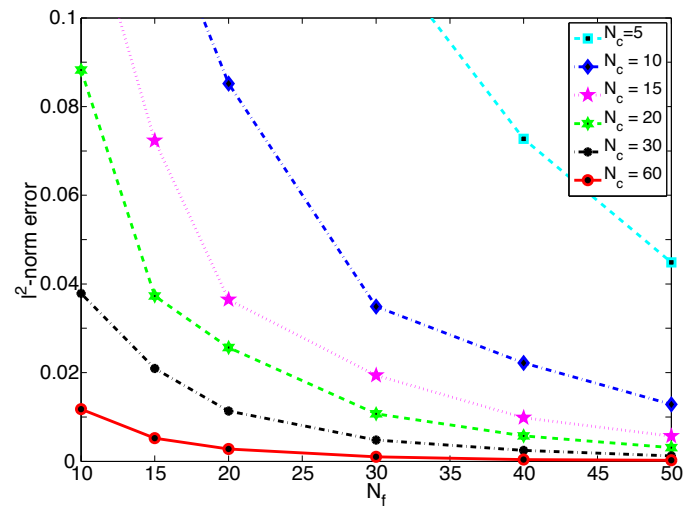

Figure 5: $l^{2}$-norm of error for 4-node basis functions (Example 2)

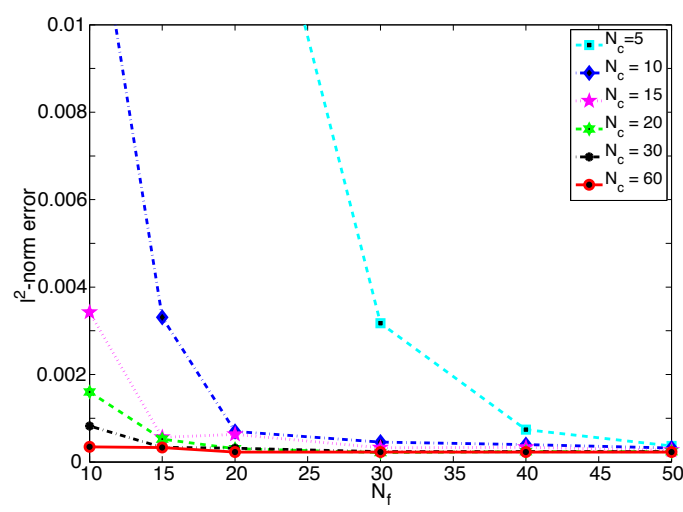

Figure 6: $l^{2}$-norm of error for 9 -node basis functions (Example 2)

Table 3 and Figure 7 show that $10 \times 10$ meshes with 9-node elements provide almost the same level of accuracy as $60 \times 60$ meshes with 4-node elements. Moreover, comparing the results of these two types of coarse meshes shows a faster convergence with increase in $N_{f}$ for the 9-node coarse mesh (Figure 7). Figure 8 provides a typical dependence of the $l^{2}$-norm of the error on the refinement of the coarse scale. Here too, for a similar refinement on the small scale, the 9-node elements provide better accuracy than the 4-node elements. Figure 9 summarizes the results discussed here in a 3D representation of the error with respect to number of elements in both the fine and coarse meshes. 


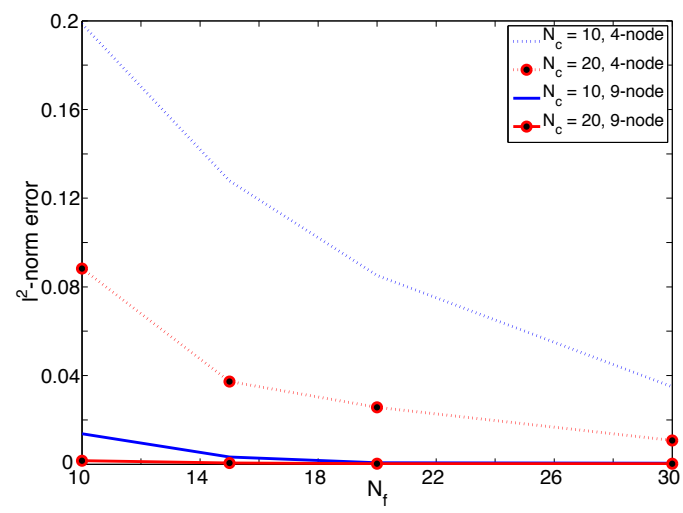

Figure 7: Dependence of $l^{2}$-norm of error on fine scale refinement (Example 2)

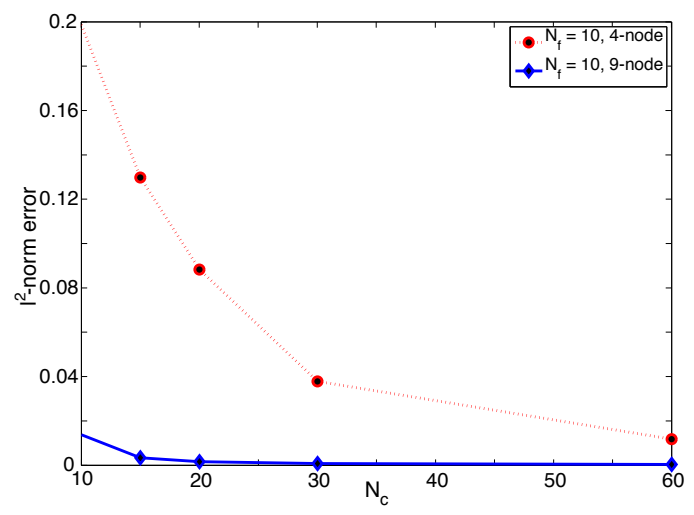

Figure 8: Dependence of $l^{2}$-norm of error on coarse scale refinement (Example 2)

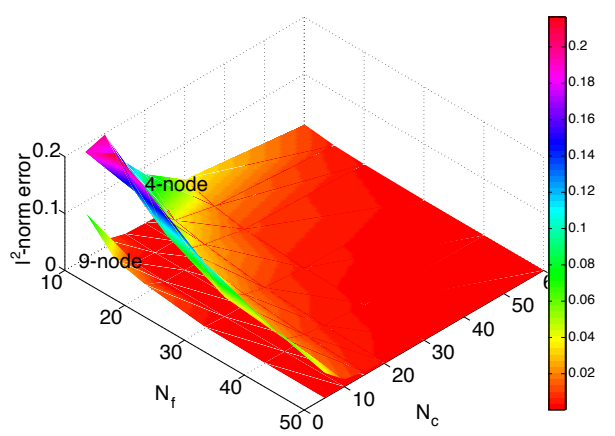

Figure 9: $l^{2}$-norm of error for 4 and 9-node elements (Example 2)
This example clearly shows that for similar coarse meshes, increasing the order of MsFEM basis functions improves the accuracy. Consequently, choosing higher order bases decreases the minimum required number of elements in the coarse mesh for achieving a certain level of accuracy. This feature is extremely valuable not only to decrease of the size of the problem, but also it indirectly uses fewer elements in the coarse mesh for problems with periodic oscillations and provides a better chance to select an appropriate mesh that matches the period of oscillations. Thus, we evaluate the bases only once and eliminate the need for parallel computation in this step.

The benefits discussed here are obtained without significant increase in the computational cost. Figure 10 depicts the dependence of the $l^{2}$-norm of the error on the analytical computational cost for similar coarse meshes, but with 4-node and 9-node elements. As shown there, using 9-node elements leads to a better accuracy while reducing the computational cost. Figure 11 shows the dependence of the error on the small scale mesh size. Note that for coarse meshes, implementing higher order elements allows for a significant reduction in the error for similar subcell sizes.

Example 3 (periodic non-separable conductivity tensor): Using similar dimensions and boundary conditions as the previous example, the conductivity tensor for this problem is given by $a(\mathbf{x})=a_{0}\left(1.5+\sin \frac{2 \pi x}{\varepsilon}\right)^{-1}+a_{0}\left(1.5+\cos \frac{2 \pi y}{\varepsilon}\right)^{-1}$, with $\varepsilon=1 / 12$ (60 oscillations in each direction over one element). Due to the non-separable function used for the conductivity tensor, we implement the standard FEM in the fine scale to evaluate the corresponding $2 \mathrm{D}$ bases. Table 4 illustrates the MsFEM solution of this problem for different discretization levels in the coarse and fine scales. These results confirm that similar to the previous problem, using higher order MsFEM bases can significantly improve the accuracy of the solution. As shown in Table 4, regardless of the refinement 


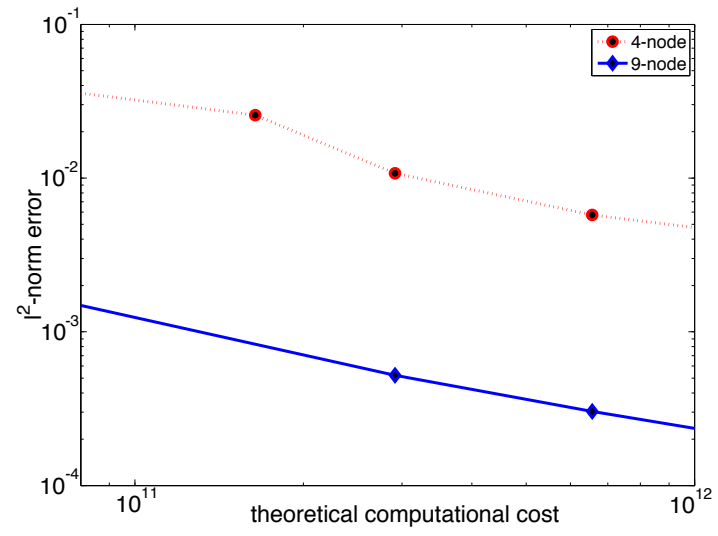

Figure 10: $l^{2}$-norm of error dependence on the computational cost (Example 2)

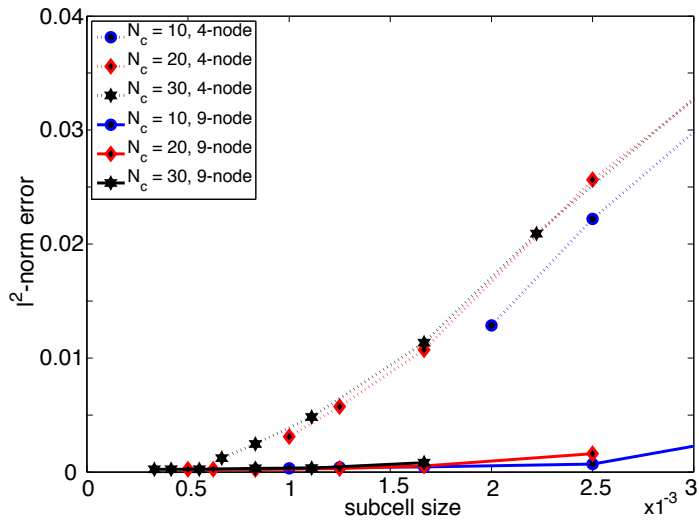

Figure 11: $l^{2}$-norm of error dependence on fine scale element size (Example 2)

in the fine scale, the accuracy reached with 12-node elements can never be achieved by modified bilinear bases. A better accuracy for the MsFEM solution with 4-node elements can only be obtained with techniques such as oversampling to reduce the resonance error, which impose a considerable extra cost to the solution. Moreover, compared to the simple proposed approach for constructing higher order elements, the upscaling approach demands a more complicated computational scheme. Figure 12 depicts the variations of the $l^{2}$-norm of the error for $N_{c}=20$.

\begin{tabular}{|c|c||c|c||c|c||c|c|}
\hline \multicolumn{2}{|c||}{ Mesh } & \multicolumn{2}{c||}{ 4-node elements } & \multicolumn{2}{c||}{$8 / 9$-node elements } & \multicolumn{2}{c|}{$12 / 16$-node elements } \\
\hline$N_{c}$ & $N_{f}$ & $E_{l^{2}}$ & $E_{\infty}$ & $E_{l^{2}}$ & $E_{\infty}$ & $E_{l^{2}}$ & $E_{\infty}$ \\
\hline \hline 10 & 10 & $2.974 \cdot 10^{-2}$ & $5.342 \cdot 10^{-2}$ & $6.219 \cdot 10^{-3}$ & $1.138 \cdot 10^{-2}$ & $4.721 \cdot 10^{-3}$ & $8.820 \cdot 10^{-3}$ \\
\hline 10 & 20 & $6.734 \cdot 10^{-3}$ & $1.230 \cdot 10^{-2}$ & $2.684 \cdot 10^{-4}$ & $4.252 \cdot 10^{-4}$ & $1.413 \cdot 10^{-4}$ & $3.110 \cdot 10^{-4}$ \\
\hline 10 & 40 & $1.769 \cdot 10^{-3}$ & $3.921 \cdot 10^{-3}$ & $1.279 \cdot 10^{-4}$ & $3.434 \cdot 10^{-4}$ & $6.954 \cdot 10^{-5}$ & $1.317 \cdot 10^{-4}$ \\
\hline 10 & 50 & $4.738 \cdot 10^{-3}$ & $4.305 \cdot 10^{-3}$ & $1.262 \cdot 10^{-4}$ & $3.427 \cdot 10^{-4}$ & $6.887 \cdot 10^{-5}$ & $1.308 \cdot 10^{-4}$ \\
\hline \hline 20 & 10 & $1.020 \cdot 10^{-2}$ & $1.834 \cdot 10^{-2}$ & $2.477 \cdot 10^{-4}$ & $4.368 \cdot 10^{-4}$ & $1.772 \cdot 10^{-4}$ & $3.170 \cdot 10^{-4}$ \\
\hline 20 & 20 & $2.217 \cdot 10^{-3}$ & $4.049 \cdot 10^{-3}$ & $1.089 \cdot 10^{-4}$ & $1.819 \cdot 10^{-4}$ & $7.969 \cdot 10^{-5}$ & $1.761 \cdot 10^{-4}$ \\
\hline 20 & 40 & $4.369 \cdot 10^{-4}$ & $1.206 \cdot 10^{-3}$ & $1.067 \cdot 10^{-4}$ & $1.777 \cdot 10^{-4}$ & $7.346 \cdot 10^{-5}$ & $1.752 \cdot 10^{-4}$ \\
\hline 20 & 50 & $6.254 \cdot 10^{-4}$ & $1.418 \cdot 10^{-3}$ & $1.067 \cdot 10^{-4}$ & $1.777 \cdot 10^{-4}$ & $7.346 \cdot 10^{-5}$ & $1.752 \cdot 10^{-4}$ \\
\hline \hline 30 & 10 & $4.162 \cdot 10^{-3}$ & $7.320 \cdot 10^{-3}$ & $5.060 \cdot 10^{-4}$ & $8.038 \cdot 10^{-4}$ & $5.161 \cdot 10^{-4}$ & $8.203 \cdot 10^{-4}$ \\
\hline 30 & 20 & $1.269 \cdot 10^{-3}$ & $2.194 \cdot 10^{-3}$ & $1.039 \cdot 10^{-4}$ & $1.365 \cdot 10^{-4}$ & $7.112 \cdot 10^{-5}$ & $1.632 \cdot 10^{-4}$ \\
\hline 30 & 40 & $4.791 \cdot 10^{-4}$ & $9.567 \cdot 10^{-4}$ & $1.037 \cdot 10^{-4}$ & $1.358 \cdot 10^{-4}$ & $7.091 \cdot 10^{-5}$ & $1.627 \cdot 10^{-4}$ \\
\hline 30 & 50 & $4.611 \cdot 10^{-4}$ & $9.228 \cdot 10^{-4}$ & $1.037 \cdot 10^{-4}$ & $1.358 \cdot 10^{-4}$ & $7.091 \cdot 10^{-5}$ & $1.627 \cdot 10^{-4}$ \\
\hline
\end{tabular}

Table 4: Example 3 (periodic nonseparable conductivity tensor). Performance of higher order elements

Example 4 (non-periodic non-separable conductivity tensor): In this last example, we use the same domain and boundary conditions as before but a different pattern (shown in Figure 13) for variations of the conductivity tensor. The results of solving this problem for two coarse meshes with different orders of basis functions are illustrated in Figures 14 and 15. The faster convergence and improvement of the accuracy of the solution is evident as we switch from first order to higher order bases. This behavior can be easily observed by comparing the results obtained using 8-node 


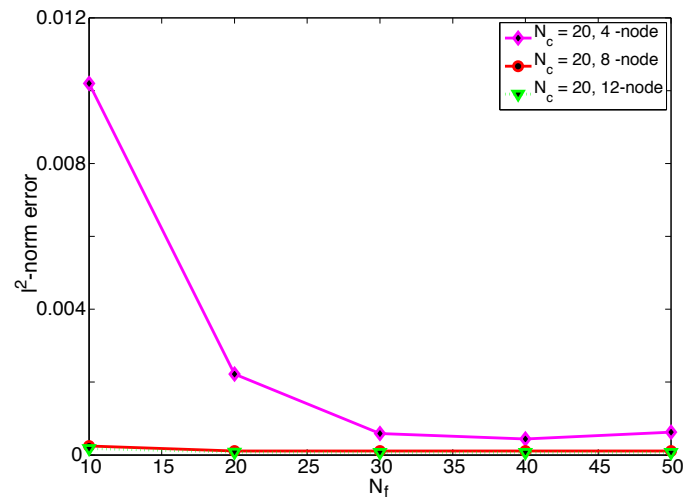

Figure 12: Performance of different order bases (Example 3)

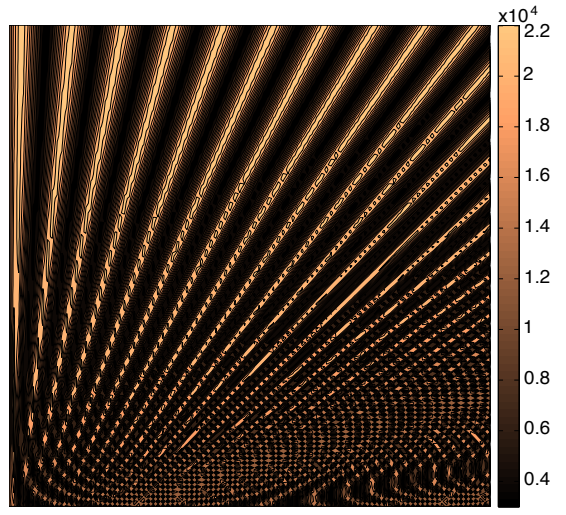

Figure 13: Oscillations in the conductivity tensor (Example 4)

elements with a $10 \times 10$ coarse mesh and those obtained with 4 -node elements on a $20 \times 20$ mesh. While the total number of degrees of freedom is similar for both cases, the solution with 8-node elements has a considerably better accuracy. Also, note from Figures 14 and 15 that refining the fine scale mesh for 4-node elements does not improve the accuracy considerably.

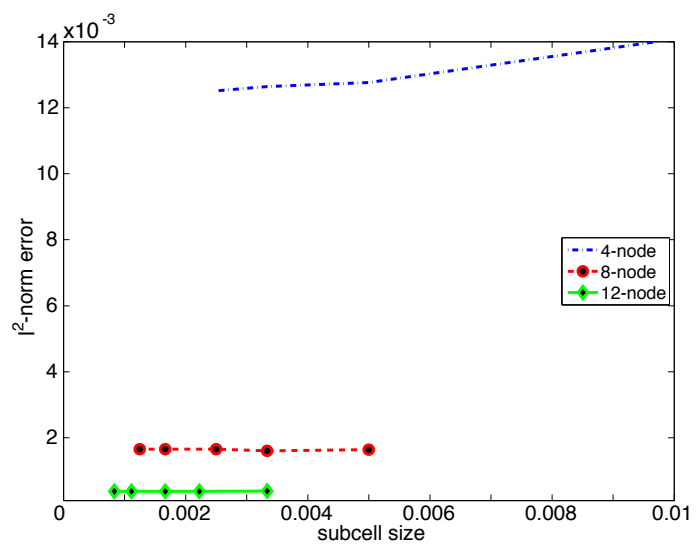

Figure 14: Variation of $l^{2}$-norm of error for a $10 \times 10$ coarse mesh (Example 4)

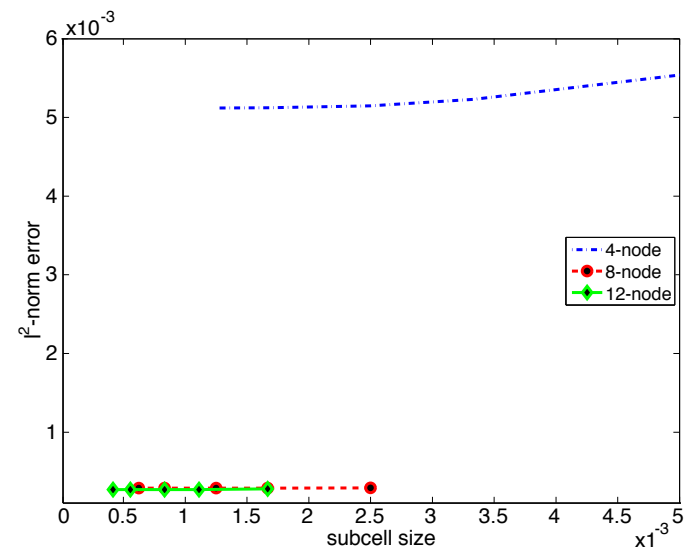

Figure 15: Variation of $l^{2}$-norm of error for a $20 \times 20$ coarse mesh (Example 4 )

\section{Conclusions}

A simple and yet effective algorithm for the construction of higher order MsFEM basis functions for second-order elliptic problems is introduced. The strengths of the method are the simplicity in the implementation and the ability to generate bases for elements with any number and organization of nodes. We showedthat using the proposed higher order bases yield a significant improvement in the accuracy compared to modified bilinear bases. Throughout the paper, the similarity between 
the proposed higher order MsFEM bases and Lagrangian shape functions in the standard FEM was employed to predict the behavior of the MsFEM and examine the correctness of results.

\section{References}

[1] K. A. Gonthier and V. Jogi. Multiscale shock heating analysis of a granular explosive. Journal of Applied Mechanics, 72(4):538-552, 2005.

[2] A. Corsini, F. Menichini, F. Rispoli, A. Santoriello, and T. E. Tezduyar. A multiscale finite element formulation with discontinuity capturing for turbulence models with dominant reactionlike terms. Journal of Applied Mechanics, 76(2), 2009.

[3] B. B. Dykaar and P. K. Kitanidis. Determination of the effective hydraulic conductivity for heterogeneous porous media using a numerical specteral approach 1. method. Water Resources Reseach, 28(4):1155-1166, 1992.

[4] R. Luce and S. Perez. A numerical upscaling method for an elliptic equation with heterogeneous tensorial coefficients. International Journal for Numerical Methods in Engineering, 54:537-556, 2002.

[5] T. Arbogast. Analysis of two-scale, locally conservative subgrid upscaling for elliptic problems. SIAM Journal on Numerical Analysis, 42:576-598, 2004.

[6] T. J. R. Hughes. Multiscale phenomena: Green's functions, the Drichlet to Neumann formulation, subgrid scale models, bubbles and the origin of the stablized methods. Computer Methods in Applied Mechanics and Engineering, 127:387-401, 1995.

[7] T. J. R. Hughes, G. R. Feijoo, L. Mazzei, and J. B. Quincy. The variational multiscale method - a paradigm for computational mechanics. Computer Methods in Applied Mechanics and Engineering, 166:3-24, 1998.

[8] I. Babuska, G. Caloz, and J. E. Osborn. Special finite element methods for a class of second order elliptic problems with rough coefficients. SIAM Journal on Numerical Analysis, 31(4):945-981, 1994.

[9] B. Engquist and Y. H. Tsai. Heterogeneous multiscale methods for stiff ordinary differential equations. Mathematics of Computations, 74(252):1707-1742, 2005.

[10] W. E, B. Engquist, and Z. Huang. Heterogeneous multiscale method: a general methodology for multiscale modeling. Condensed Matters and Material Physics, 67(9):92101-1-4, 2003.

[11] T. Y. Hou and X. H. Wu. A multiscale finite element method for elliptic problems in composite materials and porous media. Journal of Computational Physics, 134:169-189, 1997.

[12] T. Y. Hou, X. H. Wu, and Z. Cai. Convergence of a multiscale finite element method for elliptic problems with rapidly oscillating coefficients. Mathematics of Computation, 68(227):913-943, 1999.

[13] Y. R. Efendiev and H. J. Wu. Multiscale finite element method for problems with highly oscilatory. Numerische Mathematik, 90:459-486, 2002.

[14] P. Ming and X. Yue. Numerical methods for multiscale elliptic problems. Journal of Computational Physics, 214:421-445, 2006.

[15] G. Allaire and R. Brizzi. A multiscale finite element method for numerical homogenization. Multiscale Modeling \& Simulation, 4(3):790-812, 2005. 


\title{
Systematic Construction of Higher Order Bases for the Finite Element Analysis of Multiscale Elliptic Problems
}

\author{
Soheil Soghrati ${ }^{1}$ and Ilinca Stanciulescu, ${ }^{2, *}$ \\ ${ }^{1}$ University of Illinois at Urbana-Champaign, Urbana, IL 61801, U.S.A. \\ ${ }^{2}$ Rice University, Houston, TX, 77005, U.S.A. \\ ${ }^{*}$ Corresponding Author, ilinca@rice.edu
}

\begin{abstract}
We introduce a simple approach for deriving higher order basis functions for a multiscale finite element method (MsFEM) that relies on capturing the small scale features through the bases utilized in the coarse scale solution. The proposed technique for the derivation of such bases is completely systematic and the increase in the computational cost is insignificant. The implementation of higher order basis functions in the MsFEM leads to the same advantages the use of higher order Lagrangian shape functions have in the conventional finite element method. We present several numerical examples for elliptic problems with multiple scales to demonstrate the efficiency and improved characteristics of the proposed higher order basis functions.
\end{abstract}

\section{Introduction}

Second order elliptic differential equations with multiple-scale features appear in many problems in Material Science, and Engineering such as heat conduction in heterogeneous materials and flow in porous media $[10,5]$. In these problems, variations and fluctuations in material properties in the fine scale have a crucial effect on the behavior of the coarse scale. Employing the conventional finite element method (CFEM) for such problems requires resolving the small scale to yield acceptable accuracy and thus may need tens or even hundreds of millions of degrees of freedom. Consequently, the large size of the discrete problem significantly increases the computational cost. At best, it leads to very long running times but in many cases it can easily exceed the available memory capacity.

Parallel implementation alleviates limitations in computational resources but can not reduce the size of the discrete problem. To address the latter problem, several techniques were developed, e.g., based on the homogenization theory [6] or on upscaling methods $[15,2,3]$. They aim to determine effective properties of the model and then use a coarse discretization for the full system simulation. [11, 12,8] introduce a multiscale finite element method (MsFEM) for solving elliptic problems with oscillatory coefficients. The main idea of this method is to transfer the effect of the fine scale oscillations to the coarse scale by modifying the standard bilinear shape functions to some oscillatory basis functions. This method, for which we provide here a systematic technique to compute higher order bases, decouples the calculation of the basis functions in each element and 
greatly suites the parallel analysis approach. The principal difficulty of direct implementation of the MsFEM is its slow rate of convergence due to the resonance between the grid size and the physical scale of the problem. To reduce the resonance error, an oversampling technique is proposed [11] where the sampling is performed over a larger domain and interior data are used to evaluate the basis functions. Although this technique significantly reduces the error and improves the accuracy, it also increases the computational cost. We show that higher order bases in the MsFEM considerably improve the rate of convergence and the accuracy. Moreover, with the technique proposed in this paper, such improvements are achieved with insignificant increase in the computational cost.

The algorithm presented in [11] is limited to modifying standard linear or bilinear shape functions. Extension to higher order basis functions is the next step. Among previous efforts in this field we can mention the work by Allaire and Brizzi [1]. Their method is however mathematically complex and achieves the desired results with increased computational cost. By contrast, we introduce in this paper a simpler and yet generalized and accurate algorithm for constructing higher order bases in the MsFEM for elements with any desired number and configuration of nodes.

Alternative approaches for incorporating the fine scale information in the coarse scale basis functions are presented in other publications. Hughes and coworkers [13, 14] proposed a variational multiscale method (VMM) in which the solution procedure has two steps: the non-numerical solution of the fine scale and its use for the solution at the coarse scale. Babuska et al [4] used a generalized finite element method with special non-polynomial finite element bases for the approximation of second-order elliptical problems. Another general framework, the heterogeneous multiscale method (HMM), is developed by Engquist et al [9, 7]. In this approach, different scales of the model are separated by solving the problem at the macro-scale and evaluating the missing data at this level by performing a local solution on the fine scale. Reference [16] presents a comprehensive overview on recent multiscale methods and specifically the MsFEM for handling second-order elliptic problems.

The rest of the paper is organized as follows. In section 2, we present the elliptic multiscale problem and briefly explain the original MsFEM formulation in [11]. In section 3, the proposed derivation of higher order MsFEM basis functions is presented. We first introduce a systematic approach for developing basis functions for 1D problems with any desired number of nodes and then show how these 1D basis functions can be employed for constructing 2D basis functions where nodes are distributed over the element boundaries. We also introduce techniques for adding nodes inside the element and discuss the possible advantages of the resulted elements. In section four, the computational cost, accuracy and the rate of convergence of these elements are analyzed.

\section{Second Order Elliptic Problems and MsFEM}

Consider the domain $\Omega \subset \mathbb{R}^{n}$, with boundary $\Gamma=\bar{\Omega} \backslash \Omega=\Gamma_{u} \cup \Gamma_{q}$ (where Dirichlet and Neumann boundary conditions are defined). Let $\mathbf{n}$ be the outward unit normal and $\mathbf{a}(\mathbf{x})$ a positive definite tensor. The problem of interest in this study is the second-order elliptic problem

$$
\begin{array}{ccccc}
-\nabla \cdot(\mathbf{a}(\mathbf{x}) \nabla u) & = & f & \text { in } & \Omega \\
u & = & g(\mathbf{x}) & \text { on } & \Gamma_{u} \\
\mathbf{a}(\mathbf{x}) \nabla u \cdot \mathbf{n} & = & h(\mathbf{x}) & \text { on } & \Gamma_{q}
\end{array}
$$

that can model for instance heat conduction in composite materials or steady flows in porous media. 
The weak form of Eq. (2.1) is written as

$$
\text { Find } u \in \mathcal{U} \text { such that } \int_{\Omega} \mathbf{a}(\mathbf{x}) \nabla u \nabla v d \Omega=\int_{\Omega} f v d \Omega+\int_{\Gamma_{q}} g(\mathbf{x}) v d \Gamma \quad \forall v \in \mathcal{V},
$$

where $\mathcal{U}=\left\{u(\mathbf{x}),\left.u\right|_{\Gamma_{u}}=g(\mathbf{x})\right\} \subset H^{1}(\bar{\Omega})$ and $\mathcal{V}=\left\{v(\mathbf{x}),\left.v\right|_{\Gamma_{u}}=0\right\} \subset H_{0}^{1}(\bar{\Omega})$ are the solution and weighting spaces. Eq. (2.2) can be used to derive the Galerkin approximation and consequently obtain the CFEM solution of (2.1). However, such computation demands a highly-refined mesh to resolve the smallest scale of the problem and thus is extremely expensive if $\mathbf{a}(\mathbf{x})$ is heterogeneous or highly oscillatory. In contrast, the MsFEM can handle such problems through a computationally less expensive approach. The key idea is to discretize Eq. (2.1) using a coarse mesh and shape functions calculated such that they capture the small scale effects. Inside each coarse element $\Omega^{k}$ the modified shape functions $N_{i}^{(k)}$ are evaluated by solving the homogenized form of Eq. (2.1)

$$
\nabla \cdot\left(\mathbf{a}(\mathbf{x}) \nabla N_{i}^{(k)}\right)=0 \quad \text { in } \quad \Omega^{k} \subset \Omega,
$$

subject to appropriate boundary conditions. We require that the functions satisfy the Kronecker delta property at the element nodes $N_{i}^{(k)}\left(x_{j}\right)=\delta_{i j}$. Determining appropriate boundary conditions for Eq. (2.3) is not trivial; choices made here significantly influence the accuracy of the MsFEM solution. Two choices for the boundary conditions (for four node 2D elements) are proposed in [11]. The first is to consider linear functions, i.e., restrictions to the boundaries of the standard bilinear shape functions. This choice does not lead to satisfactory results since it neglects the oscillations in the fine scale over the boundaries of the coarse mesh. A second option is proposed, where the effect of oscillations in the fine scale properties is included by obtaining the solution $N_{i}^{\Gamma}$ of the reduced form of Eq. (2.3) over each boundary of the course element $\frac{d}{d x_{\Gamma}}\left(\mathbf{a}_{\Gamma}\left(x_{\Gamma}\right) \frac{d N_{i}^{\Gamma}}{d x_{\Gamma}}\right)=0$ on $\Gamma_{i}^{k}=\partial \Omega^{k}$, subject to $N_{i}^{\Gamma}\left(x_{j}\right)=\delta_{i j}$. In the special case where the conductivity tensor is a separable function, $\mathbf{a}(\mathbf{x})=\mathbf{a}_{1}(x) \mathbf{a}_{2}(y)$, the $2 \mathrm{D}$ basis functions can be computed directly through the tensor product of the boundary conditions. Oscillatory boundary conditions provide more accurate results. However, resonance between the solutions at different scales remains an important issue and is a source of error that can not be effectively removed by refining the fine scale mesh. The interested reader can find a detailed description of the MsFEM including the estimation of the resonance error in [11, 12].

\section{Extension to Higher Order Elements}

In this section we introduce a general approach for evaluating the MsFEM shape functions for higher order elements with different number of nodes. We aim to preserve some of the properties of the Lagrangian shape functions in the higher order MsFEM bases. The approach we propose not only avoids heavy mathematical derivation and the use of homogenization theory in computing the MsFEM bases, but also provides more insight over the features of these bases. A direct extension of the procedure explained in section 2 can be followed. A CFEM problem is solved over the coarse elements subject to appropriate boundary conditions. The boundary conditions associated with Eq. (2.3) determine how well the characteristics of the fine scale are captured in the basis and thus transferred to the solution at the coarse scale. In order to achieve an acceptable accuracy, we need to 
incorporate the fine scale information in the boundary conditions of higher order bases. To achieve this goal, we first devise an approach for evaluating higher order bases for 1D problems, which are very effective as boundary conditions for the $2 \mathrm{D}$ problem. We then introduce several techniques for constructing the basis for $2 \mathrm{D}$ elements with different number and distribution of nodes.

\subsection{Higher Order Bases for 1D Problems}

Consider the 1D elliptic problem

$$
\frac{d}{d x}\left(a(x) \frac{d u}{d x}\right)=f \quad \forall x \in[0, L] .
$$

Constructing two-node bases for the MsFEM solution of this problem using the method outlined in section 2 is quite straightforward: they are the solutions of $\frac{d}{d x}\left(a(x) \frac{d u}{d x}\right)=0 \quad \forall x \in[0, L]$ subject to $N_{i}^{k}\left(x_{j}\right)=\delta_{i j}$ boundary conditions. In order to extend this algorithm to higher order bases, we require that the basis functions are $C^{0}$-continuous and satisfy the partition of unity, i.e., $\sum_{i=1}^{n} N_{i}^{k}=1$.

Before outlining an effective algorithm for generating higher order bases, we discuss some characteristics of the Lagrangian shape functions. Our goal is to obtain similar properties for the MsFEM higher order basis functions. Unlike linear shape functions, higher order Lagrangian shape functions do not satisfy the homogenized form of a second order elliptic equation. Similarly, we see no reason to require higher order multiscale bases to satisfy this equation. The $2^{\text {nd }}$ order Lagrangian shape functions are constructed through the linear combination of $1^{\text {st }}$ order Lagrangian shape functions and a parabolic function. This parabolic function can be the solution of a particular instance of Eq. (3.1) $\left(a(x)=a_{0}\right)$, subject to a constant $f=\bar{f}$ and zero essential boundary conditions. Similarly, multiscale bases for three-node elements, $N_{i}^{(3)}$, can be constructed by linear combination of two-node basis functions, $N_{e}^{(2)}$, and the solution $N_{p}$ of Eq. (3.1) with $f=1$ and subject to zero essential boundary conditions. The following notation convention is used: $e$ and $i$ are the nodes of the two-node and of the higher order element, respectively. We obtain for a three-node element $N_{i}^{(3)}=\delta_{e i} N_{e}^{(2)}+\alpha_{i} N_{p}$, where $\alpha_{i}$ are determined such that $\left.N_{i}^{(3)}\right|_{\xi_{j}}=\delta_{i j}$. Note that this property is satisfied by construction at the end nodes and only the condition at the middle node is non-trivial:

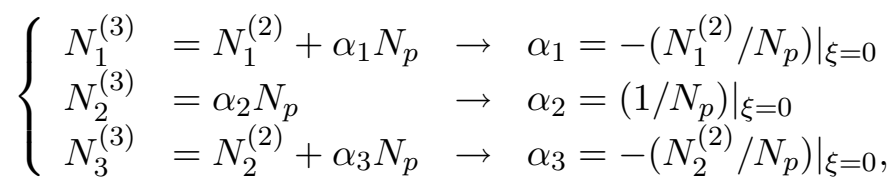

where $\xi \in[-1,1]$ are the natural coordinates. Figure 1 compares second order standard Lagrangian shape functions with the multiscale basis functions for a conductivity tensor with five oscillations per element. $a(x)=2000(1.3+\sin 400 \pi x)(1.3+\cos (400 \pi x+2 \pi / 5))$. Note that the resulted basis converges to the standard Lagrangian basis as the oscillations in the properties of the fine scale are damped out. The proposed approach for evaluating the basis functions of three-node elements can be easily extended to even higher order bases. For instance, the four-node basis functions are constructed by the linear combination of the two-node basis functions $N_{e}^{(2)}$ and $N_{p 1}$ and $N_{p 2}$, the solutions of Eq. (3.1) with $f=1$ and $f=\xi$, subject to zero essential boundary conditions: 
$N_{i}^{(4)}=\delta_{e i} N_{e}^{(2)}+\alpha_{i} N_{p 1}+\beta_{i} N_{p 2}$. The coefficients $\alpha_{i}$ and $\beta_{i}$ are chosen such that the functions satisfy the Kronecker delta property at the element nodes, $N_{i}^{(4)}\left(\xi_{j}\right)=\delta_{i j}$, where $\xi_{j} \in\left\{ \pm 1, \pm \frac{1}{3}\right\}$.
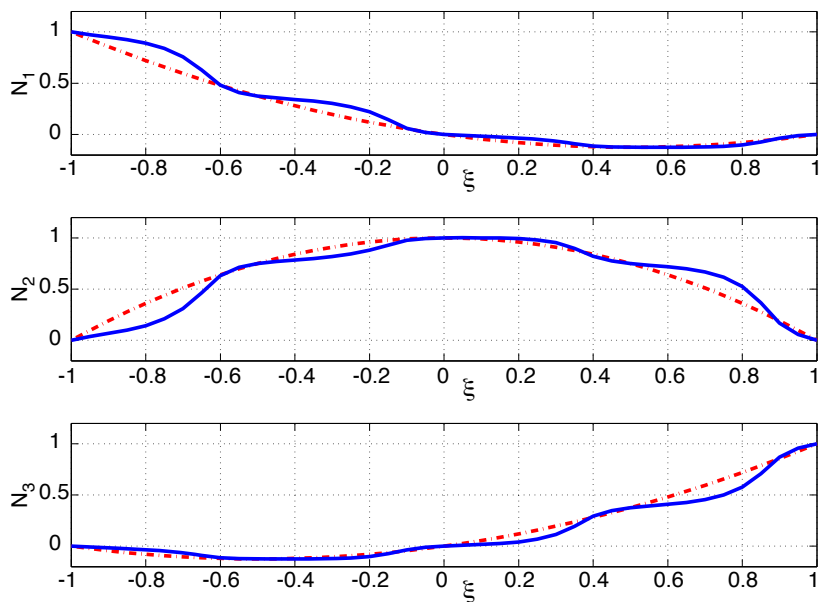

Figure 1: Multiscale (solid lines) and Lagrangian (dotted lines) shape functions

\subsection{Higher Order Bases for 2D Problems}

The extension of the algorithm described in section 2 to higher order $2 \mathrm{D}$ bases is not difficult provided that good choices are made for the boundary conditions of the coarse domains. We use the 1D bases as boundary conditions for the local domain (i.e., for an element of the coarse mesh) and construct higher order bases for elements with nodes distributed only over the boundaries. The boundary conditions for an 8-node element and the resulted bases are shown in figure 2 .
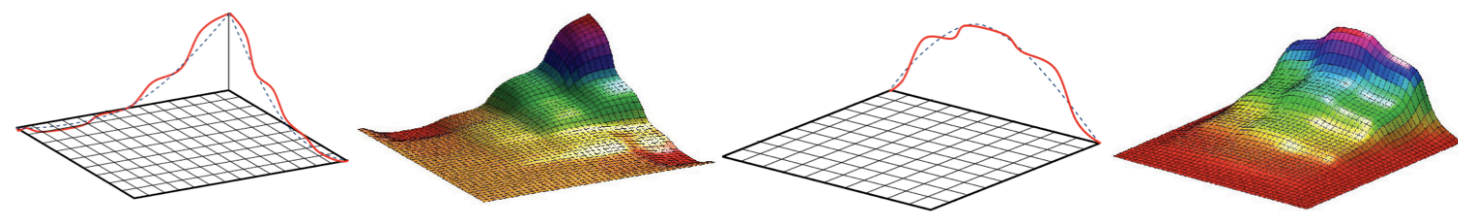

Figure 2: Boundary conditions and resulted MsFEM basis functions for an 8-node element

To construct basis functions for elements with interior nodes, we solve Eq. (2.1) subject to zero boundary conditions and appropriate polynomial right-hand-side functions. For instance, for a 9-node element, we determine the solution $N_{p}$ of $-\nabla \cdot(\mathbf{a}(\mathbf{x}) \nabla u)=1$ in $\Omega^{k}$ subject to $u=0$ on $\partial \Omega^{k}$. We then construct the higher order basis functions as linear combinations of $N_{p}$, with the basis functions $N_{e}^{(8)}$ of the corresponding Serendipity-type 8-node element $N_{e}^{(9)}=\delta_{e i} N_{e}^{(8)}+\alpha_{i} N_{p}$.

If the conductivity tensor is a separable function in the Euclidian space, i.e., $\mathbf{a}(\mathbf{x})=\mathbf{a}_{1}(x) \mathbf{a}_{2}(y)$, we can use a computationally less expensive method: 2D shape functions are obtained from the tensor product of $1 \mathrm{D}$ bases evaluated over the boundaries of the coarse element (figure 3.) The 
numerical evaluation and the tensor product yield different basis functions. When evaluating numerically, we are looking for bases that satisfy the homogenized equation for serendipity-type elements, while the result of the tensor product does not satisfy this equation. On the other hand, as the oscillations in the fine scale of the problem are damped out, the resulted bases by the tensor product approach converge to serendipity shape functions while the CFEM bases do not. Results in section 4 show that both have similar level of accuracy in problems with separable conductivity tensors. The efficiency of the tensor product approach makes it better suited to such problems.

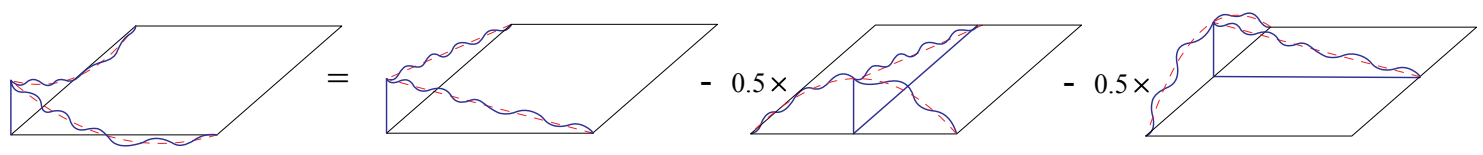

Figure 3: Construction of MsFEM basis functions through the tensor product of 1D bases

\section{Numerical Experiments}

In this section, we study the performance of the proposed higher order bases through several numerical tests. We compare results of the MsFEM using modified bilinear basis functions with results obtained with higher order bases and report the convergence. The reference solutions are obtained by CFEM with extremely refined meshes that resolve the small scale.

Example 1: For 1D problems, the MsFEM yields the same results as the CFEM, provided that the product of the number of elements in the fine and course meshes, $N_{f} N_{c}$, is equal to the number of elements utilized in the CFEM solution. However, using higher order basis functions for solving 1D problems sheds more light on the behavior of these bases and offers additional insight in analyzing the MsFEM algorithm. For these reason, we first present a $1 D$ example: $\frac{d}{d x}\left(a_{0}\left(1.3+\sin \left(\frac{2 \pi x}{\varepsilon}\right)\right)\left(1.3+\cos \left(\frac{2 \pi x}{\varepsilon}+\frac{2 \pi}{5}\right)\right) \cdot \frac{d u}{d x}\right)=-1000 \forall x \in[0, L]$, where $u(0)=u(L)=0$, and $\varepsilon=0.025$ characterizes the small scale properties (40 oscillations per unit length of the domain). The length of the domain is $L=5$ and the number of elements in the coarse mesh is $N_{c}=100$.

Table 1 shows the performance of the MsFEM solution. The two cases correspond to utilizing linear and second-order Lagrangian shape functions in the fine scale. The values of the $l^{2}$-norm of the error for this problem are exactly the same for all coarse scale bases (2, 3 or 4 -node). At the first glance, this might indicate the low performance of higher order bases: despite the additional cost imposed to the solution, higher order bases do not improve the convergence of the MsFEM. This behavior is easily explained: MsFEM is the generalization of the CFEM through modification of shape functions to include the small scale information. Accordingly, the behavior of the bases in the MsFEM is similar to that of the Lagrangian shape functions of the same order in the CFEM for a similar problem but with a constant conductivity tensor. Employing any Lagrangian shape functions for solving a 1D elliptic problem with constant conductivity tensor through the CFEM yields super-convergent results for the nodal values. Similarly, different order of bases in the MsFEM yield the same solution at the common nodes of the coarse mesh.

The main advantage of higher order 1D bases appears in problems with more than two scales. The order of the Lagrangian shape functions used in the solution at the small scale has a crucial 


\begin{tabular}{|c|c|c|c|}
\hline \multicolumn{2}{|c|}{$1^{\text {st }}$ order } & \multicolumn{2}{c|}{$2^{\text {nd }}$ order } \\
\hline$N_{f}$ & $\|E\|_{l^{2}}$ & $N_{f}$ & $\|E\|_{l^{2}}$ \\
\hline \hline 100 & $9.163 \cdot 10^{-4}$ & 10 & $8.723 \cdot 10^{-3}$ \\
\hline 200 & $2.287 \cdot 10^{-4}$ & 20 & $6.218 \cdot 10^{-4}$ \\
\hline 300 & $1.012 \cdot 10^{-4}$ & 30 & $9.914 \cdot 10^{-5}$ \\
\hline 400 & $5.660 \cdot 10^{-5}$ & 40 & $3.179 \cdot 10^{-5}$ \\
\hline 500 & $3.589 \cdot 10^{-5}$ & 50 & $1.266 \cdot 10^{-5}$ \\
\hline 600 & $2.467 \cdot 10^{-5}$ & 60 & $5.692 \cdot 10^{-6}$ \\
\hline 700 & $1.789 \cdot 10^{-5}$ & 70 & $2.689 \cdot 10^{-6}$ \\
\hline 800 & $1.350 \cdot 10^{-5}$ & 80 & $1.226 \cdot 10^{-6}$ \\
\hline
\end{tabular}

Table 1: MsFEM Results (Example 1)

\begin{tabular}{|c|c|c|c|c|c|}
\hline \multicolumn{2}{|c|}{ Two-scaled } & \multicolumn{3}{c|}{ Three-scaled solution } & \\
\hline$N_{f}$ & $\|E\|_{l^{2}}$ & $N_{f 1}$ & $N_{f 2}$ & $\|E\|_{l^{2}}$ & Nodes \\
\hline \hline 100 & $9.163 \cdot 10^{-4}$ & 10 & 5 & $5.219 \cdot 10^{-4}$ & 10000 \\
\hline 200 & $2.287 \cdot 10^{-4}$ & 20 & 5 & $8.220 \cdot 10^{-5}$ & 20000 \\
\hline 300 & $1.012 \cdot 10^{-4}$ & 30 & 5 & $5.012 \cdot 10^{-5}$ & 30000 \\
\hline 400 & $5.660 \cdot 10^{-5}$ & 40 & 5 & $2.815 \cdot 10^{-5}$ & 40000 \\
\hline 500 & $3.589 \cdot 10^{-5}$ & 25 & 10 & $\mathbf{9 . 4 1 1} \cdot 10^{-6}$ & 50000 \\
\hline 600 & $2.467 \cdot 10^{-5}$ & 30 & 10 & $7.215 \cdot 10^{-6}$ & 60000 \\
\hline 700 & $1.789 \cdot 10^{-5}$ & 35 & 10 & $5.076 \cdot 10^{-6}$ & 70000 \\
\hline 800 & $1.350 \cdot 10^{-5}$ & 40 & 10 & $3.128 \cdot 10^{-6}$ & 80000 \\
\hline
\end{tabular}

Table 2: Two and three scaled solutions (Example 1)

effect on the accuracy. The reason can be traced back to the effect of the oscillations in the small scale properties which prevents the CFEM from yielding super-convergent results. In this case, as shown in Table 1, using $2^{\text {nd }}$ order Lagrangian shape functions for the solution at the small scale yields a better accuracy per computation cost than linear functions. For instance, 50 second-order elements in the small scale provide better accuracy than 800 linear elements (the size of the resulted stiffness matrix is therefore reduced by a factor of 8 when second order elements are used.)

The same characteristic can be used to reduce the size of the problem. For instance, we can solve the current problem in three scales, using linear shape functions in the finest scale, threenode bases in the small scale and 2-node bases in the coarse mesh. In this case, we expect to achieve more accurate results compared to the solution obtained if only two scales with linear and modified linear shape functions are used. Table 2 confirms the expectation: while the total number of degrees of freedom is kept constant in each row, the three-scaled solution described above yields more accurate results. Note that using three scales for solving this problem is only a choice for the numerical solution and does not correspond to the physical scales of the problem.

Example 2: We now demonstrate the efficiency of utilizing

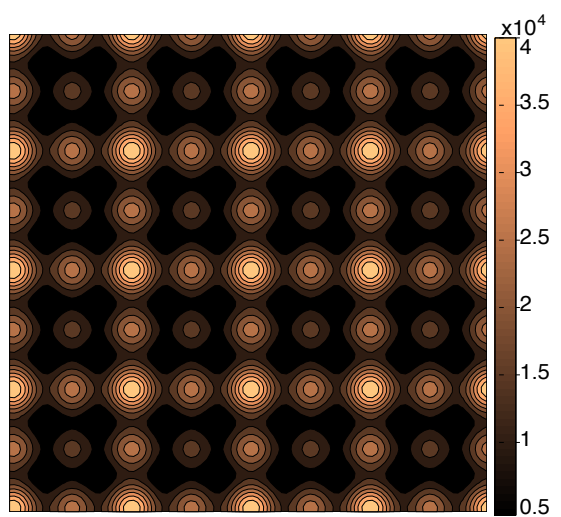

Figure 4: Oscillations in the conductivity tensor (Example 2) higher order bases for solving a $2 \mathrm{D}$ problem with periodic oscillations in the small scale properties. We solve Eq. (2.1) for $a(x)=a_{0}\left(1.3+\sin \frac{2 \pi x}{\varepsilon}\right)\left(1.3+\sin \frac{2 \pi y}{\varepsilon}\right)\left(1.3+\cos \left(\frac{2 \pi x}{\varepsilon}+\frac{2 \pi}{5}\right)\right)$ $\left(1.3+\cos \left(\frac{2 \pi y}{\varepsilon}+\frac{2 \pi}{5}\right)\right)$ and $f=-1000$ over a $5 \times 5$ square domain, subject to zero essential boundary conditions. $\varepsilon=1 / 12$ is used: it corresponds to 60 oscillations in each direction over the domain (figure 4). The MsFEM solutions using 4 and 9node elements (using the tensor product approach) are reported in Table 3 and the convergence in $l^{2}$-norm error is illustrated in figures 5 and 6 . Note that for clarity the range of error in the two graphs differs by a factor of 10 . The accuracy of the MsFEM solution is limited by the size of the coarse mesh (independent of the resolution of the discretization in the fine scale). The use of $10 \times 10$ or $20 \times 20$ coarse mesh with modified bilinear shape functions does not provide results with a satisfactory 
level of accuracy (the $l^{2}$-norms of the error even for extremely refined mesh in the fine scale) are approximately 10 times larger than the error obtained using 9-node basis functions (Table 3).

\begin{tabular}{|c|c||c|c||c|c|}
\hline \multicolumn{2}{|c||}{ Mesh } & \multicolumn{2}{c||}{ 4-node elements } & \multicolumn{2}{c|}{ 9-node elements } \\
\hline$N_{c}$ & $N_{f}$ & $E_{l^{2}}$ & $E_{\infty}$ & $E_{l^{2}}$ & $E_{\infty}$ \\
\hline 10 & 10 & $1.986 \cdot 10^{-1}$ & $3.550 \cdot 10^{-1}$ & $1.372 \cdot 10^{-2}$ & $2.452 \cdot 10^{-2}$ \\
\hline 10 & 20 & $8.521 \cdot 10^{-2}$ & $1.525 \cdot 10^{-1}$ & $6.958 \cdot 10^{-4}$ & $9.512 \cdot 10^{-4}$ \\
\hline 10 & 30 & $3.489 \cdot 10^{-2}$ & $6.110 \cdot 10^{-2}$ & $4.561 \cdot 10^{-4}$ & $7.308 \cdot 10^{-4}$ \\
\hline 10 & 40 & $2.220 \cdot 10^{-2}$ & $4.010 \cdot 10^{-2}$ & $3.961 \cdot 10^{-4}$ & $6.149 \cdot 10^{-4}$ \\
\hline 10 & 50 & $1.286 \cdot 10^{-2}$ & $2.343 \cdot 10^{-2}$ & $3.186 \cdot 10^{-4}$ & $5.851 \cdot 10^{-4}$ \\
\hline 20 & 10 & $8.825 \cdot 10^{-2}$ & $1.576 \cdot 10^{-1}$ & $4.599 \cdot 10^{-4}$ & $7.484 \cdot 10^{-4}$ \\
\hline 20 & 20 & $2.564 \cdot 10^{-2}$ & $4.588 \cdot 10^{-2}$ & $3.030 \cdot 10^{-4}$ & $5.538 \cdot 10^{-4}$ \\
\hline 20 & 30 & $1.073 \cdot 10^{-2}$ & $1.924 \cdot 10^{-2}$ & $2.146 \cdot 10^{-4}$ & $3.958 \cdot 10^{-4}$ \\
\hline 20 & 40 & $5.744 \cdot 10^{-3}$ & $1.029 \cdot 10^{-2}$ & $2.343 \cdot 10^{-4}$ & $4.098 \cdot 10^{-4}$ \\
\hline 20 & 50 & $3.109 \cdot 10^{-3}$ & $5.720 \cdot 10^{-3}$ & $2.342 \cdot 10^{-4}$ & $4.088 \cdot 10^{-4}$ \\
\hline
\end{tabular}

\begin{tabular}{|c|c||c|c||c|c|}
\hline \multicolumn{2}{|c||}{ Mesh } & \multicolumn{2}{c||}{ 4-node elements } & \multicolumn{2}{c|}{ 9-node elements } \\
\hline$N_{c}$ & $N_{f}$ & $E_{l^{2}}$ & $E_{\infty}$ & $E_{l^{2}}$ & $E_{\infty}$ \\
\hline 30 & 10 & $3.786 \cdot 10^{-2}$ & $6.772 \cdot 10^{-2}$ & $8.219 \cdot 10^{-4}$ & $1.420 \cdot 10^{-3}$ \\
\hline 30 & 20 & $1.134 \cdot 10^{-2}$ & $2.028 \cdot 10^{-2}$ & $3.155 \cdot 10^{-4}$ & $4.968 \cdot 10^{-4}$ \\
\hline 30 & 30 & $4.820 \cdot 10^{-3}$ & $8.720 \cdot 10^{-3}$ & $2.380 \cdot 10^{-4}$ & $4.153 \cdot 10^{-4}$ \\
\hline 30 & 40 & $2.463 \cdot 10^{-3}$ & $4.381 \cdot 10^{-3}$ & $2.350 \cdot 10^{-4}$ & $4.149 \cdot 10^{-4}$ \\
\hline 30 & 50 & $1.206 \cdot 10^{-3}$ & $2.341 \cdot 10^{-3}$ & $2.350 \cdot 10^{-4}$ & $4.149 \cdot 10^{-4}$ \\
\hline 60 & 10 & $1.173 \cdot 10^{-2}$ & $2.094 \cdot 10^{-2}$ & $3.475 \cdot 10^{-4}$ & $6.247 \cdot 10^{-4}$ \\
\hline 60 & 20 & $2.781 \cdot 10^{-3}$ & $5.038 \cdot 10^{-3}$ & $2.266 \cdot 10^{-4}$ & $4.087 \cdot 10^{-4}$ \\
\hline 60 & 30 & $1.031 \cdot 10^{-3}$ & $1.793 \cdot 10^{-3}$ & $2.266 \cdot 10^{-4}$ & $4.087 \cdot 10^{-4}$ \\
\hline 60 & 40 & $3.909 \cdot 10^{-4}$ & $7.074 \cdot 10^{-4}$ & $2.266 \cdot 10^{-4}$ & $4.087 \cdot 10^{-4}$ \\
\hline 60 & 50 & $2.549 \cdot 10^{-4}$ & $4.277 \cdot 10^{-4}$ & $2.266 \cdot 10^{-4}$ & $4.087 \cdot 10^{-4}$ \\
\hline
\end{tabular}

Table 3: Example 2 (separable conductivity tensors). Performance of higher order elements

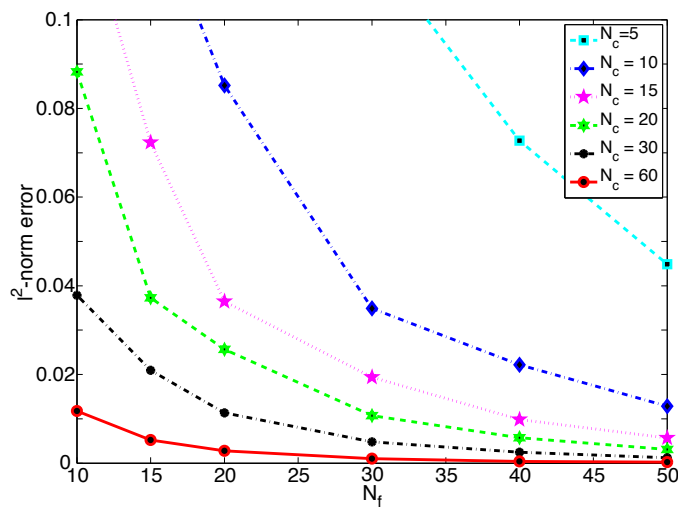

Figure 5: $l^{2}$-norm of error for 4-node basis functions (Example 2)

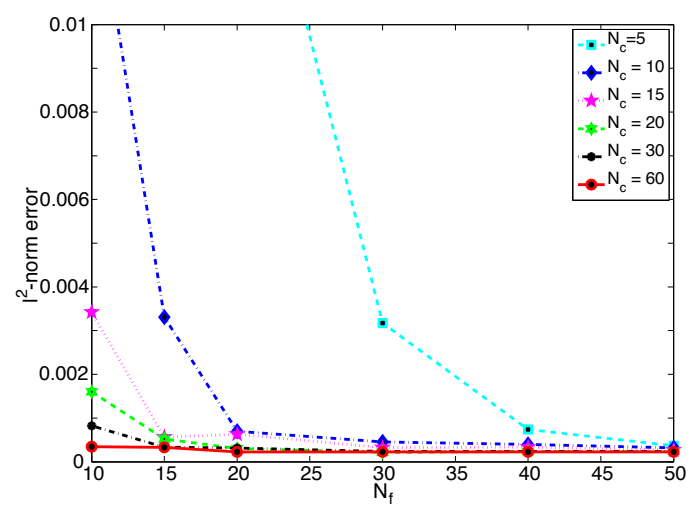

Figure 6: $l^{2}$-norm of error for 9-node basis functions (Example 2)

Table 3 and figure 7 show that $10 \times 109$-node elements provide almost the same level of accuracy as $60 \times 604$-node elements. Moreover, comparing the results of these two types of coarse meshes shows a faster convergence with increase in $N_{f}$ for the 9 -node coarse mesh (figure 7 ). Figure 8 provides a typical dependence of the $l^{2}$-norm of the error on the refinement of the coarse scale. Here too, for a similar refinement on the small scale, the 9-node elements provide better accuracy than the 4 -node elements. Figure 9 summarizes the results.

This example clearly shows that for similar coarse meshes, increasing the order of the bases in the MsFEM improves the accuracy. Consequently, choosing higher order bases decreases the minimum required number of elements in the coarse mesh for achieving a certain level of accuracy. This feature is extremely valuable not only for the direct decrease of the size of the problem but also indirectly by demanding fewer elements in the coarse mesh for problems with periodic oscillations and providing a better chance to select an appropriate mesh that matches the period of the oscillations. Thus, we evaluate the basis only once and eliminate the need for parallel computation in this step. 


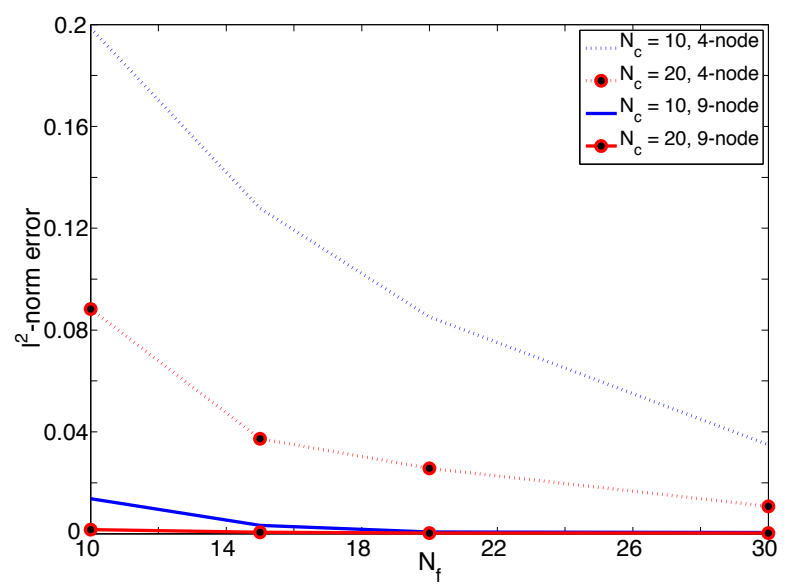

Figure 7: Dependence of the $l^{2}$-norm of error on the fine scale refinement (Example 2)

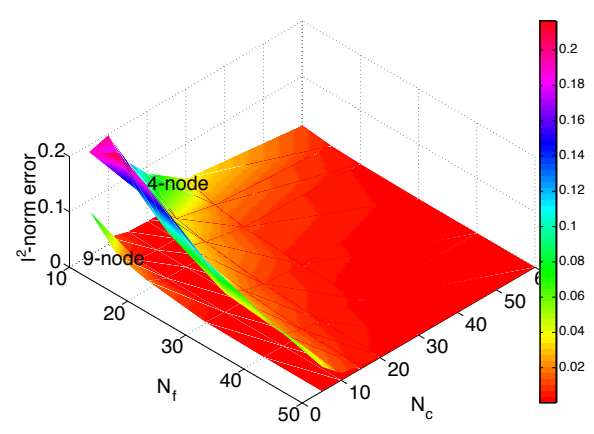

Figure 9: $l^{2}$-norm of error for 4 and 9-node elements (Example 2)

Example 3 In this example we solve a 2D problem with a nonseparable periodic conductivity tensor that requires the use of the CFEM approach in the small scale to construct the $2 \mathrm{D}$ bases. The oscillations of the conductivity tensor in Eq. (2.1) are expressed as $a(x)=\frac{a_{0}}{1.5+\sin \frac{2 \pi x}{\varepsilon}}+\frac{a_{0}}{1.5+\cos \frac{2 \pi x}{\varepsilon}}$, with $\varepsilon=1 / 12$ (60 oscillations in each direction over one element). Table 4 shows the results of the MsFEM solution of this problem for different discretization levels in the coarse and fine scales. These results confirm that one of the advantages of using higher order bases in the MsFEM algorithm lies in improving the accuracy of the solution through decreasing the resonance effect. For the current example, regardless of the refinement in the fine scale, the accuracy reached with 12-node bases can never be achieved by modified bilinear bases. Better accuracy of the MsFEM solution with 4-node elements can only be obtained with techniques such as oversampling that impose a considerable extra cost to the solution. Moreover, compared to the simple proposed approach for constructing higher order elements, the upscaling technique in the MsFEM demands a more complicated computational scheme. Figure 12 depicts the variations of the $l^{2}$-norm error for $N_{c}=20$.

Example 4. In this last example, we show the performance of the higher order bases for prob- 


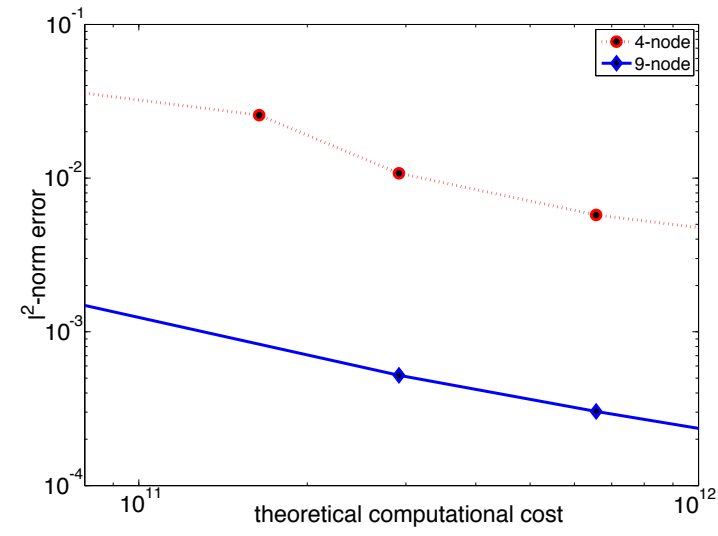

Figure 10: $l^{2}$-norm of error dependence on the computational cost (Example 2)

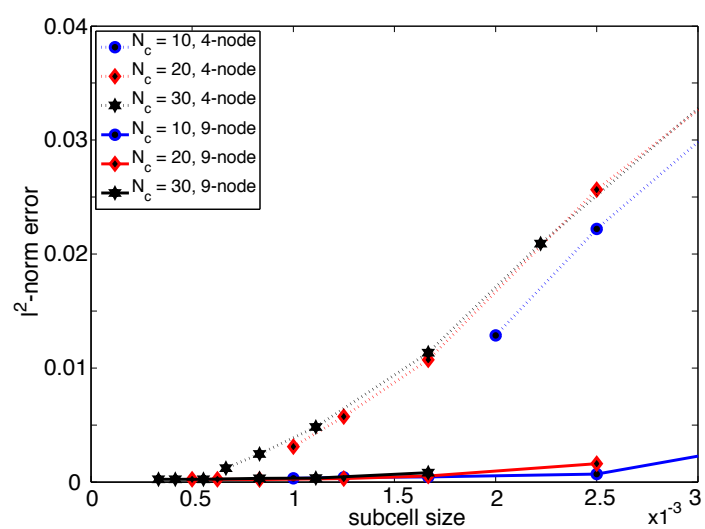

Figure 11: $l^{2}$-norm of error dependence on fine scale element size (Example 2)

\begin{tabular}{|c|c||c|c||c|c||c|c|}
\hline \multicolumn{2}{|c||}{ Mesh } & \multicolumn{2}{c||}{ 4-node elements } & \multicolumn{2}{c||}{$8 / 9$-node elements } & \multicolumn{2}{c|}{$12 / 16$-node elements } \\
\hline$N_{c}$ & $N_{f}$ & $E_{l^{2}}$ & $E_{\infty}$ & $E_{l^{2}}$ & $E_{\infty}$ & $E_{l^{2}}$ & $E_{\infty}$ \\
\hline \hline 10 & 10 & $2.974 \cdot 10^{-2}$ & $5.342 \cdot 10^{-2}$ & $6.219 \cdot 10^{-3}$ & $1.138 \cdot 10^{-2}$ & $4.721 \cdot 10^{-3}$ & $8.820 \cdot 10^{-3}$ \\
\hline 10 & 20 & $6.734 \cdot 10^{-3}$ & $1.230 \cdot 10^{-2}$ & $2.684 \cdot 10^{-4}$ & $4.252 \cdot 10^{-4}$ & $1.413 \cdot 10^{-4}$ & $3.110 \cdot 10^{-4}$ \\
\hline 10 & 30 & $8.878 \cdot 10^{-4}$ & $3.561 \cdot 10^{-3}$ & $5.535 \cdot 10^{-4}$ & $9.326 \cdot 10^{-4}$ & $5.241 \cdot 10^{-4}$ & $7.310 \cdot 10^{-4}$ \\
\hline 10 & 40 & $1.769 \cdot 10^{-3}$ & $3.921 \cdot 10^{-3}$ & $1.279 \cdot 10^{-4}$ & $3.434 \cdot 10^{-4}$ & $6.954 \cdot 10^{-5}$ & $1.317 \cdot 10^{-4}$ \\
\hline 10 & 50 & $4.738 \cdot 10^{-3}$ & $4.305 \cdot 10^{-3}$ & $1.262 \cdot 10^{-4}$ & $3.427 \cdot 10^{-4}$ & $6.887 \cdot 10^{-5}$ & $1.308 \cdot 10^{-4}$ \\
\hline \hline 20 & 10 & $1.020 \cdot 10^{-2}$ & $1.834 \cdot 10^{-2}$ & $2.477 \cdot 10^{-4}$ & $4.368 \cdot 10^{-4}$ & $1.772 \cdot 10^{-4}$ & $3.170 \cdot 10^{-4}$ \\
\hline 20 & 20 & $2.217 \cdot 10^{-3}$ & $4.049 \cdot 10^{-3}$ & $1.089 \cdot 10^{-4}$ & $1.819 \cdot 10^{-4}$ & $7.969 \cdot 10^{-5}$ & $1.761 \cdot 10^{-4}$ \\
\hline 20 & 30 & $5.894 \cdot 10^{-4}$ & $1.123 \cdot 10^{-3}$ & $1.070 \cdot 10^{-4}$ & $1.783 \cdot 10^{-4}$ & $7.346 \cdot 10^{-5}$ & $1.752 \cdot 10^{-4}$ \\
\hline 20 & 40 & $4.369 \cdot 10^{-4}$ & $1.206 \cdot 10^{-3}$ & $1.067 \cdot 10^{-4}$ & $1.777 \cdot 10^{-4}$ & $7.346 \cdot 10^{-5}$ & $1.752 \cdot 10^{-4}$ \\
\hline 20 & 50 & $6.254 \cdot 10^{-4}$ & $1.418 \cdot 10^{-3}$ & $1.067 \cdot 10^{-4}$ & $1.777 \cdot 10^{-4}$ & $7.346 \cdot 10^{-5}$ & $1.752 \cdot 10^{-4}$ \\
\hline \hline 30 & 10 & $4.162 \cdot 10^{-3}$ & $7.320 \cdot 10^{-3}$ & $5.060 \cdot 10^{-4}$ & $8.038 \cdot 10^{-4}$ & $5.161 \cdot 10^{-4}$ & $8.203 \cdot 10^{-4}$ \\
\hline 30 & 20 & $1.269 \cdot 10^{-3}$ & $2.194 \cdot 10^{-3}$ & $1.039 \cdot 10^{-4}$ & $1.365 \cdot 10^{-4}$ & $7.112 \cdot 10^{-5}$ & $1.632 \cdot 10^{-4}$ \\
\hline 30 & 30 & $6.648 \cdot 10^{-4}$ & $1.123 \cdot 10^{-3}$ & $1.037 \cdot 10^{-4}$ & $1.358 \cdot 10^{-4}$ & $7.091 \cdot 10^{-5}$ & $1.627 \cdot 10^{-4}$ \\
\hline 30 & 40 & $4.791 \cdot 10^{-4}$ & $9.567 \cdot 10^{-4}$ & $1.037 \cdot 10^{-4}$ & $1.358 \cdot 10^{-4}$ & $7.091 \cdot 10^{-5}$ & $1.627 \cdot 10^{-4}$ \\
\hline 30 & 50 & $4.611 \cdot 10^{-4}$ & $9.228 \cdot 10^{-4}$ & $1.037 \cdot 10^{-4}$ & $1.358 \cdot 10^{-4}$ & $7.091 \cdot 10^{-5}$ & $1.627 \cdot 10^{-4}$ \\
\hline
\end{tabular}

Table 4: Example 3 (periodic nonseparable conductivity tensor). Performance of higher order elements

lems with non-periodic and nonseparable variations in the fine scale properties. For this purpose, we use the same domain and boundary conditions as before but a different pattern (shown in Figure 13) for the variations of the conductivity tensor. The results of solving this problem for two coarse meshes with different orders of basis functions are illustrated in Figures 14 and 15. The faster convergence and improvement of the accuracy of the solution is evident as we switch from first order to higher order bases. This behavior can be easily observed by comparing the results obtained using 8 -node elements with a $10 \times 10$ coarse mesh and and those obtained with 4-node elements and a $20 \times 20$ mesh. While the total number of degrees of freedom is of the same order, the solution with 8-node elements achieves a considerably better accuracy. Also, note from Figures 14 and 15 that refining the fine scale mesh for 4-node elements does not improve the accuracy considerably; 


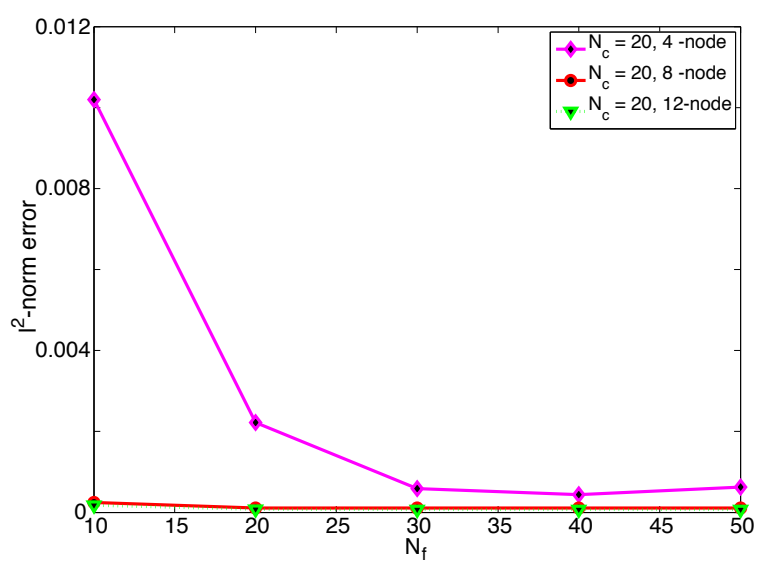

Figure 12: Performance of different order bases (Example 3)

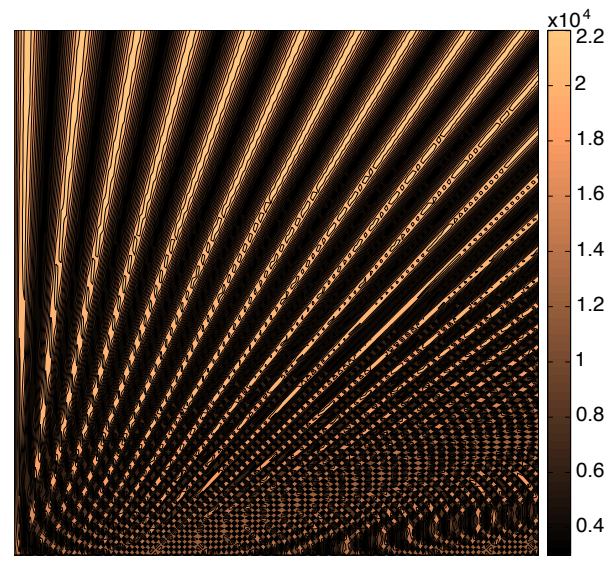

Figure 13: Oscillations in the conductivity tensor (Example 4)

instead, increasing the order of the bases is much more effective.

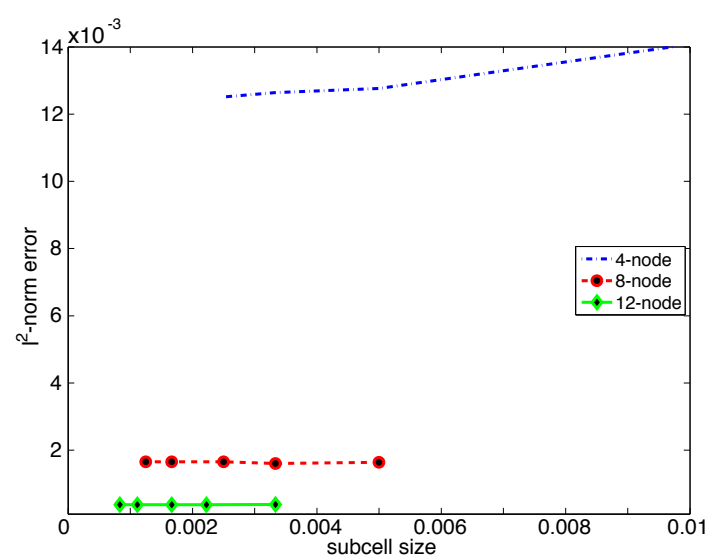

Figure 14: Variation of the $l^{2}$-norm of error for a $10 \times 10$ coarse grid (Example 4)

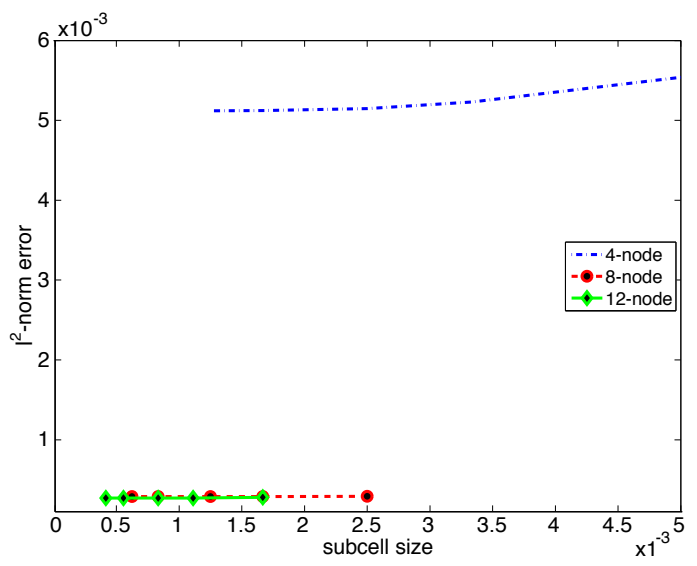

Figure 15: Variation of the $l^{2}$-norm of error for a $20 \times 20$ coarse grid (Example 4 )

\section{Conclusions}

A simple and yet effective algorithm for the construction of higher order MsFEM basis functions for second order elliptic problems is proposed. The strengths of the method are its simplicity in implementation and its ability to generate bases for elements with any number and organization of nodes. We demonstrate the significant improvement in accuracy that higher order bases provide compared to the modified bilinear shape functions. Throughout this paper, the similarity between the proposed higher order MsFEM bases and the higher order Lagrangian shape functions in CFEM is used as the main tool to predict the behavior of the MsFEM and examine the correctness of results. 


\section{References}

[1] G. Allaire and R. Brizzi. A multiscale finite element method for numerical homogenization. Multiscale Modeling \& Simulation, 4(3):790-812, 2005.

[2] T. Arbogast. Analysis of two-scale, locally conservative subgrid upscaling for elliptic problems. SIAM Journal on Numerical Analysis, 42:576-598, 2004.

[3] T. Arbogast and K. J. Boyd. Subgrid upscaling and mixed multiscale finite elements. SIAM Journal on Numerical Analysis, 44:1150-1171, 2006.

[4] I. Babuska, G. Caloz, and J. E. Osborn. Special finite element methods for a class of second order elliptic problems with rough coefficients. SIAM Journal on Numerical Analysis, 31(4):945-981, 1994.

[5] A. Corsini, F. Menichini, F. Rispoli, A. Santoriello, and T. E. Tezduyar. A multiscale finite element formulation with discontinuity capturing for turbulence models with dominant reactionlike terms. Journal of Applied Mechanics, 76(2), 2009.

[6] B. B. Dykaar and P. K. Kitanidis. Determination of the effective hydraulic conductivity for heterogeneous porous media using a numerical specteral approach 1. method. Water Resources Reseach, 28(4):1155-1166, 1992.

[7] W. E, B. Engquist, and Z. Huang. Heterogeneous multiscale method: a general methodology for multiscale modeling. Condensed Matters and Material Physics, 67(9):92101-1-4, 2003.

[8] Y. R. Efendiev and H. J. Wu. Multiscale finite element method for problems with highly oscilatory. Numerische Mathematik, 90:459-486, 2002.

[9] B. Engquist and Y. H. Tsai. Heterogeneous multiscale methods for stiff ordinary differential equations. Mathematics of Computations, 74(252):1707-1742, 2005.

[10] K. A. Gonthier and V. Jogi. Multiscale shock heating analysis of a granular explosive. Journal of Applied Mechanics, 72(4):538-552, 2005.

[11] T. Y. Hou and X. H. Wu. A multiscale finite element method for elliptic problems in composite materials and porous media. Journal of Computational Physics, 134:169-189, 1997.

[12] T. Y. Hou, X. H. Wu, and Z. Cai. Convergence of a multiscale finite element method for elliptic problems with rapidly oscillating coefficients. Mathematics of Computation, 68(227):913-943, 1999.

[13] T. J. R. Hughes. Multiscale phenomena: Green's functions, the Drichlet to Neumann formulation, subgrid scale models, bubbles and the origin of the stablized methods. Computer Methods in Applied Mechanics and Engineering, 127:387-401, 1995.

[14] T. J. R. Hughes, G. R. Feijoo, L. Mazzei, and J. B. Quincy. The variational multiscale method - a paradigm for computational mechanics. Computer Methods in Applied Mechanics and Engineering, 166:3-24, 1998.

[15] R. Luce and S. Perez. A numerical upscaling method for an elliptic equation with heterogeneous tensorial coefficients. International Journal for Numerical Methods in Engineering, 54:537-556, 2002.

[16] P. Ming and X. Yue. Numerical methods for multiscale elliptic problems. Journal of Computational Physics, 214:421-445, 2006. 